\title{
AN OPTIMAL SIMULATION OF COUNTER MACHINES*
}

\author{
PAUL M. B. VITÁNYI†
}

\begin{abstract}
Each multicounter machine can be simulated by an oblivious one-head tape unit in real-time, using logarithmic space. The solution uses redundant symmetric number representation and implicit recursion. It represents a new positional representation for (vectors of) the integers.
\end{abstract}

Key words. counter machines, multicounter machines, real-time simulation by one-tape Turing machines, redundant symmetric number representation, implicit recursion, counting, coding

1. Introduction. The idea of counting, that is, adding or subtracting a unit from any given number, to obtain another one, is the substrate of arithmetic if not of all of mathematics. Thus, it is frequently necessary in computing to maintain many counts simultaneously, while the only information we want to extract at any time is the set of currently zero counts. The process of storing several integer counts, each count independently being incremented or decremented by a unit in each step, governed by the current input and the set of zero counts, is abstracted and formalized in the notion of a multicounter machine. Such machines have numerous connections with both theoretical issues and more or less practical applications. It is of considerable interest, for many questions, to implement multicounter machines as efficiently as possible. We shall show that counting is basically simple, in the computational complexity sense of the word, by demonstrating that each multicounter machine can be simulated in real-time by an oblivious one-head tape unit using minimal storage space. Since the presented implementation is optimal in all commonly considered resources at once, the two decade old quest for better simulations of multicounter machines by Turing machines is finalized in one stroke.

Doing arithmetic presupposes number representations. Different representations are better suited to different arithmetical operations. All of arithmetic can be performed by multicounter machines. Because we shall simulate a múlticounter machine by a one-head tape unit, we need to straightforwardly represent a vector of integers as a linear string. No known representation for single integers allows the counter steps to be performed by an oblivious one-head tape unit without unbounded time loss in between simulated steps. Neither does any known representation, for pairs of integers, allow the counter steps to be performed by a one-head tape unit, oblivious or not, without unbounded time loss in between simulated steps. To achieve our objective, we in effect have to develop a new representation, with the required properties, for vectors of integers.

Multicounter machines and Turing machines. For the present purpose, machines are viewed as transducers, that is, as abstract storage devices connected to input and output terminals. Thus we consider a machine as hidden in a black box with input and output terminals. Consequently, the presented simulation results concern the inputoutput behavior of black boxes and are independent of input-output conventions, or whether we want to recognize or to compute. The abstract storage structure embodied by a $k$-counter machine $(k-\mathrm{CM})$ consists of a finite control connected to an input and

\footnotetext{
${ }^{*}$ Received by the editors February 8,1983 . A preliminary exposition of this work appeared in the Proceedings of the 14th ACM Symposium on Theory of Computing (STOC) held in San Francisco, California, May 5-7, 1982. The present work is registered at the Mathematical Centre as IW 216/82.

† Centre for Mathematics and Computer Science, Kruislaan 413, 1098 SJ Amsterdam, The Netherlands.
} 
an output terminal, and $k$ counters each capable of containing any integer. The states of the finite control are partitioned into polling and autonomous states. (Here we can assume without loss of generality that all states are polling states.) At the start of a computation the finite control of the $k-\mathrm{CM}$ is in a designated initial state and all counters are set to zero. A step in a $k$-CM computation is uniquely determined by the state of the finite control, by the symbol scanned at the input terminal if the state is a polling state and the set of counters which contain zero. The action at that step consists of independently altering the contents of each counter by $-1,0$, or +1 , changing the state of the finite control and producing an, possibly empty, output string. Thus the machine effects a transduction from input strings to output strings. If you will, the input and output may be thought of as written on input and output tapes, on which the resident access pointers (heads) are steered by the finite control. The steering commands issued can be viewed as part of the output. Above we closely followed the formulation in [2] where also a more precise definition can be found. For the more standard concept of multitape Turing machines consult [2], [6]. Note that, for us, a one-tape Turing machine consists of a finite control connected to an input and output terminal, and a single head storage tape. A one-head tape unit is a one-tape Turing machine.

Simulation. A machine $A$ simulates a machine $B$ in time $T(n)$ if, for all $n>0$, the input/output behavior of $B$ during the first $n$ steps, the atomic inputs and outputs ordered according to their occurrences in time, is exactly mimicked by $A$ within the first $T(n)$ steps. That is, if for every input sequence $i_{1}, i_{2}, \cdots$ read from the input terminal: (i) the output sequences written to the output terminals by $A$ and $B$ are the same, and (ii) if $t_{1} \leqq t_{2} \leqq \cdots \leqq t_{k} \leqq t_{k+1} \cdots$ are the steps at which $B$ reads or writes a symbol, then there are corresponding steps $t_{1}^{\prime} \leqq t_{2}^{\prime} \leqq \cdots \leqq t_{k}^{\prime} \leqq t_{k+1}^{\prime} \cdots$ at which $A$ reads or writes the same symbols, and $t_{i}^{\prime} \leqq T\left(t_{i}\right)$ for all $i \geqq 1$. For a linear time simulation it is required that $T(n) \in O(n)$; for a simulation with constant delay that $t_{n+1}^{\prime}-t_{n}^{\prime} \leqq$ $c\left(t_{n+1}-t_{n}\right)$ for some fixed constant $c$ and all $n$; for a real-time simulation that $T(n)=n$. It is well known that a constant delay simulation can always be sped up to a real-time one, but not a linear time simulation in general. We use simulation in the above strong sense of on-line simulation [6] throughout.

Obliviousness. A Turing machine is oblivious if the movements of the storage tape heads are fixed functions of time independent of the particular inputs to the machine. Many problems seem inherently oblivious: the usual algorithms for computing the four main arithmetic operations, a table look-up by sequential search, can easily be programmed obliviously without sacrificing worst case time efficiency. Other tasks like binary search or sorting are, it appears, nonoblivious in nature. For many purposes, there are excellent reasons to restrict attention to oblivious computations [6], [7]. Here we show yet another, more heuristic, motive for doing so. Viz., restriction of the considered model of computation to its oblivious version may shift the emphasis in the problem to be solved, from one difficulty to a completely different one, thus directing us to a solution. Whereas the difficulty in real-time simulating $k$-counter machines by $k^{\prime}$-tape Turing machines, $k^{\prime}<k$, stems from the fact that $k^{\prime}<k$, the same problem with the simulating machine restricted to its oblivious version knows as difficulty but the obliviousness of the simulating device alone.

For suppose we can simulate some abstract storage device $S$ in time $T(n)$ by an oblivious Turing machine $M$. Then we can also simulate a collection of $k$ copies of $S$, say $S_{1}, S_{2}, \cdots, S_{k}$, interacting through a common finite control, by dividing all storage tapes of $M$ into $k$ tracks, each of which is a duplicate of the corresponding former tape, and by an appropriate modification of $M$ 's finite control. The same head move- 
ments of the resulting machine $M^{\prime}$ can now do the same job, on each of the $k$ collections of tracks, as they formerly did on the collection of tapes of the original machine $M$. So the resources used by $M^{\prime}$ are, apart from sizes of finite control and alphabets, the same as those used by $M$. In particular this holds for time and storage complexity. Therefore the following two statements are equivalent:

(i) We can simulate an abstract storage device $S$ by an oblivious Turing machine $M$ in time $T(n)$ and storage $S(n)$.

(ii) For each $k>0$ we can simulate a collection of $k$ copies of $S$, interacting through a common finite control, by an oblivious Turing machine $M^{\prime}$ in time $T(n)$ and storage $S(n)$, where $M^{\prime}$ has the same tape/head constellation as $M$. We are in particular interested in the following specialization of the above maxim.

Define the quintessential counter $S$ as a 1-CM with input commands "add $\delta$ ", $\delta \in\{-1,0,1\}$. At each step $S$ reads an input command from the input terminal, modifies the stored count in the obvious way, and outputs either "count equal zero" or "count unequal zero" in concordance with the current state of affairs.

PROPOSIrION 1. If we can real-time simulate the quintessential counter $S$ by an oblivious one-head tape unit then we can real-time simulate each multicounter machine by an oblivious one-head tape unit (which for each multicounter machine makes the same head movements as a function of time alone).

Background. Counter machines are relatively old devices in computer science. Unrestricted 2-counter machines were shown to be as powerful as Turing machines in [5]. Subsequently the efficiency of implementations on Turing machines was investigated. On linear arrays, as formalized by Turing machines, the use of a tally representation for each count either requires a separate access pointer (storage tape head) per count or unbounded update time in between simulated steps. Curiously, even with the use of a separate pointer for each count, binary representations also require unbounded update time, although minimal storage space. This sorry state of affairs was improved in [1], [2] which both presented linear array simulations using minimal space, while [1] eliminated the unbounded update time at the cost of retaining all access pointers and [2] eliminated all access pointers but one at the cost of retaining unbounded update time. Thus, [2] exhibited the classic linear time/logarithmic space simulation of multicounter machines by one-tape Turing machines. Efforts to reduce this simulation to a real-time one using a fixed number of storage heads failed, but did produce some weaker problems. For the Origin Crossing Problem, where the task is to recognize the set of sequences of unit basis vectors in $k$-space, $k \geqq 1$, which leave from and end in the origin, an ingenious solution by a real-time one-tape Turing machine was constructed in [1]. The result implies that each $k$-counter machine can be real-time simulated by a $k$-tape Turing machine in logarithmic space, $k \geqq 1$. Next in difficulty comes the Axis Crossing Problem, where the task is to recognize the set of sequences of unit basis vectors in $k$-space, which leave from the origin and end in one of the $(k-1)$-dimensional hyperplanes with one zero coordinate, $k>1$. For no $k>1$, a real-time solution on but a $(k-1)$-tape Turing machine was found, for the $k$ dimensional Axis Crossing Problem, after its proposal in [2].

In [8] we made the linear time/logarithmic space one-tape solution of [2] oblivious, retaining the same resource bounds. This is a matter of some significance, since by its nature an oblivious Turing machine is usually far slower than a nonoblivious one. For example, each oblivious multitape Turing machine needs $n \log n$ steps to simulate $n$ steps of a single pushdown store, although an oblivious 2-tape Turing machine can achieve this bound [6]. For oblivious one-tape Turing machines the lower bound on this simulation time increases, perhaps, up to $n^{2}$. Due to the compact way the counts 
can be stored, the situation for counter machines was somewhat better. In [7, Cor. 2] it was shown how to simulate each multitape Turing machine, using at most $S(n)$ storage in $n$ steps, by an oblivious 2-tape Turing machine in $n \log S(n)$ steps and $S(n)$ storage. So the previously best simulation of multicounter machines, by combining [2] and [7], yielded a $n \log \log n$ time and $\log n$ storage simulation by an oblivious 2-tape Turing machine. Since the thrust of [8] was to achieve fast low-cost combinational logic networks implementing multicounter machines, as an expedient intermediate next result a real-time simulation by, basically, a linear iterative array with a restricted amount of oblivious local rewriting was proposed. Although not very elegant, this intermediate model served its purpose in yielding an optimal implementation of multicounter machines on combinational logic networks and, perhaps more important, the ideas embodied in the method suggest the approach to the final simulator presented here.

Outline of the paper. The objective is to construct an oblivious one-head tape unit capable of simulating any multicounter machine in real-time. In $\S 2$ a stylized version of such a simulator is exhibited and shown to work. This version, one of many which are possible on the basic underlying principles, is chosen because it is at once amenable to short rigorous proofs of validity and achieves, it seems, the utmost frugality of machinery. To a large extent this gain is obtained at a cost of loss of intuition as to how and why it does what it is supposed to do. To counterbalance this expository defect, we insert some informal comments. The reader may also follow the genesis of the result by consulting [8] and the earlier version in the STOC Proceedings. In $\S 3$ we enlarge on the optimality of the result, its connection with number representations, and on additional fruit borne.

2. The simulation. After some vain attempts to real-time simulate multicounter machines by Turing machines with a fixed number of tapes, one gets the feeling that, anyway, a real-time simulation by an oblivious one-head tape unit is out of the question. In the event, intuition is wrong; but let us informally consider the matter in some more detail. It quickly becomes apparent that updating a count, in real-time on an oblivious machine, requires a redundancy in notation which seems to make a simultaneous real-time check for zero impossible. To achieve the latter, we allow only encodings of integers such that an integer is zero iff the scanned position of the encoding (the "first" position, so to speak) shows this uniquely. Since the head motion is supposed to be oblivious we must, roughly speaking, update each "initial" $\Omega(\log i)$ length segment (situated around the head) of the encoded integer within each interval of $i$ steps, for all $i \geqq 1$. While moving the head to update longer segments of code in an oblivious manner, we may have actually stored small counts which may reach zero during this motion. So the machine has to simultaneously shift and update smaller segments of code, while updating larger segments of code, and so on recursively down to the smallest segments. Such considerations force compact encodings, and, apart from giving us some feel for what behavior is necessarily involved in a simulation as desired, they show that the integer representation used must be positional in nature.

Outline of the simulation. The simulation splits naturally into two parts. First we introduce a redundant binary representation for the integers, and formulate certain minimal requirements for real-time maintaining the representation of the stored integer under the counter operations. These requirements consist in a fixed strategy, of accessing constant length segments of this representation, for all input streams. Second, we construct an oblivious one-head tape unit capable of implementing these requirements in real-time. 
The current count of the quintessential counter, as figuring in Proposition 1, is stored on the single tape in a (garbled form of) redundant binary representation, with marked most significant nonzero digit and leading distinguished blank symbols. As a consequence of preserving some invariants, the stored count equals zero iff the "first" position of the representation is a blank. Since this first position shall reside in the finite control of the simulator, that situation is instantly recognized.

Hence the problem is solved, if we can real-time update the representation of the current count while preserving the invariants. In the chosen representation it suffices to update each segment of the $2 i$ th through $(2 i+3)$ th position of the representation at least once within each interval of $3^{i}$ consecutive steps, for all $i \geqq 0$, while also processing the current input commands, by an update of the first two positions, in each step. Intuitively speaking, the timing allows us to propagate carries and borrows (negative carries) fast enough. Although there is a considerable freedom about how to implement the required datamovement on an oblivious one-head tape unit, we choose for frugality in attendant machinery and minimal bit compression (that is, a small storage tape alphabet). Therefore, we divide the representation into blocks of two digits each, and store the first three blocks in the finite control. Each digit of the representation residing on the tape is tagged with an opening or a closing bracket, viz. the first digit of a block with an opening bracket and the second one with a closing bracket. To access each segment of the $2 i$ th through $(2 i+3)$ th digits of the representation at least once in every interval of $3^{i}$ steps, we develop a method of recursively transporting the digits of block $j$, from one side of the combination of the first $i$ blocks to the other side, back and forth, for all $i, j, 1 \leqq i<j$. This transport, which entails moving the total combination of the first $i$ blocks, in its turn supplies the necessary motion for the combination of the first $i+1$ blocks, while it also allows the single head to access blocks $i+2$ and $i+3$ within the timing constraints. The single head, without being able to determine the positional index of the scanned digits (since there will be all in all but four tags, viz. two types of opening brackets and two types of closing brackets), preserves a topology which allows it to single out and update due segments. The net effect will be that, for all $i$ simultaneously, the combination of the first $i$ blocks acts like a very fat head, moving slower the greater $i$ is, but fast enough to do the same job to blocks $i+j$ as the head itself does to blocks $j$, for all $i, j \geqq 1$.

On notation. To be able to express and prove the subsequent constructions, it is convenient to introduce some notation first. The objects operated upon are linear arrays or strings of symbols from a finite alphabet. Arrays can be finite or one-way infinite. In a one-way infinite array $A[0: \infty], A[0]$ is the first element and $A[i]$ is the $(i+1)$ th element, $i \geqq 0 . A[i: j]$ denotes the $(j-i+1)$-length subarray consisting of the $(i+1)$ th through $(j+1)$ th elements, $0 \leqq i \leqq j$. The concatenation $A[i: j] A[j+1: k]$ equals $A[i: k], 0 \leqq i \leqq j<k$, and we identify $A[i: i]$ with $A[i], i \geqq 0$. Finite arrays are treated similarly. Arrays are operated upon by functions from arrays to arrays. Since these functions shall be partial we introduce the undefined array $\varnothing$. By definition, for any array $A, \varnothing A=A \varnothing=\varnothing$. The undefined array should be distinguished from the empty array $\varepsilon$ for which by definition, for any array $A, \varepsilon A=A \varepsilon=A$. Mappings from arrays to arrays are defined in terms of length preserving functions from finite arrays to finite arrays. If a function $P$ maps an array $S$ to an array $S^{\prime}$, with $S, S^{\prime}$ finite and of equal length, then we write $P: S \mapsto S^{\prime}$. By definition $P: \varnothing \mapsto \varnothing$ for all functions $P$. Functions induce relations amongst one-way infinite arrays in essentially two ways. In the first type of relation $\stackrel{\rho}{\Leftrightarrow}$ the argument of $Q$ determines integers $i, j, i \leqq j$, and for all arrays $A[0: \infty], A^{\prime}[0: \infty]$ if $P: A[i: j] \mapsto A^{\prime}[i: j]$, for a function $P$ associated with $Q$, 
$A^{\prime}[0: i-1]=A[0: i-1]$ and $A^{\prime}[j+1: \infty]=A[j+1: \infty]$, then $A \stackrel{O}{\Rightarrow} A^{\prime}$. In this case, clearly $\stackrel{\rho}{\Rightarrow}$ is a function from one-way infinite arrays to one-way infinite arrays. In the second type of relation $\stackrel{P}{\Leftrightarrow}, P$ is a function, and $A \stackrel{P}{\Leftrightarrow} A^{\prime}$ if $A=S_{1} S S_{2}, A^{\prime}=S_{1} S^{\prime} S_{2}$ and $P: S \mapsto S^{\prime}$. It will be shown that all such relations of the second type we consider are also functions when restricted to a set of well formed arrays. In both cases, if for some $\stackrel{O}{\Leftrightarrow}$ and some array $A$, there is no $A^{\prime} \neq \varnothing$ such that $A \stackrel{\circ}{\Leftrightarrow} A^{\prime}$, then $A \stackrel{\circ}{\Leftrightarrow} \varnothing$. Considering the relation $\stackrel{\rho}{\Leftrightarrow}$ amongst arrays as rewriting, the rewriting shall thus be proved to be always monogenic, that is, if $A \stackrel{Q}{\Leftrightarrow} A^{\prime}$ and $A \stackrel{O}{\Leftrightarrow} A^{\prime \prime}$ then $A^{\prime \prime}=A^{\prime}$. We compose functions $P_{1}, P_{2}, \cdots, P_{n}$ to a function $P$, or decompose or expand a function $P$ into a sequence of constituent functions $P_{1}, P_{2}, \cdots, P_{n}$ as follows. If for some $P, P_{1}, \cdots, P_{n}$ and all arrays $A$ there exist arrays $A_{1}, A_{2}, \cdots, A_{n}$ such that $A \stackrel{P}{\Rightarrow} A_{n}$ and $A \stackrel{P_{1}}{\Leftrightarrow} A_{1} \stackrel{P_{2}}{\Leftrightarrow} A_{2} \cdots \stackrel{P_{n}}{\Rightarrow} A_{n}$ then $P=P_{1} ; P_{2} ; \cdots ; P_{n}$. The function composition operator ";" denotes sequential rewriting from left to right. Whenever necessary, we denote the value of an array $A$ at time $t, t \geqq 0$, by $A^{t}$ and $A^{0}$ is the initial array. We dispense with the superscript if $t$ is understood or when we view $A$ as a variable.

Main objective. We concentrate on real-time simulating the quintessential counter of Proposition 1 by an oblivious one-head tape unit.

2.1. An integer representation. Consider a positional base 2 notation for representing the integers, which may be called redundant symmetric binary, using the digits $-2,-1,0,1,2$. So the integer $c$ represented by $c_{0} c_{1} c_{2} \cdots c_{m}, c_{i} \in\{-2,-1,0,1,2\}$, equals $\sum_{i=0}^{m} c_{i} 2^{i}$. Such a representation is binary because of the weight of digits in distinct positions, symmetric because of the used digits, and redundant since each integer has infinitely many representations, even without leading nonsignificant zeros. To represent the stored integer count on a, potentially infinite, linear tape we essentially use a restricted version of this representation, with a marked most significant nonzero digit and distinguished leading nonsignificant zeros. Let $\Delta=\{-2,-1,0,1,2\}$ and $\bar{\Delta}=$ $\{-\overline{2},-\overline{1}, \overline{0}, \overline{1}, \overline{2}\}$. The barred digits have the same value as their nonbarred counterparts, $\bar{\Delta}-\{\overline{0}\}$ is reserved for the most significant nonzero digit, and " $\overline{0} "$ ", called blank, is reserved for the nonsignificant zeros. Let $\Sigma=\Delta U \bar{\Delta}$ and let code: $\mathbb{Z} \rightarrow 2^{\Sigma^{\infty}}$ be a function of the integers into the power set of $\Sigma^{\infty}$, where $\Sigma^{\infty}$ is the set of one-way infinite strings over $\Sigma$. The function code satisfies restrictions (A)-(D) below, for all $c_{0} c_{1} \cdots c_{i} \cdots \in$ $\operatorname{code}(c), c \in \mathbb{Z}$.

Separation of a finite significant initial segment and nonsignificant zeros:

$$
\begin{aligned}
\exists i \geqq 0\left[c_{i}=\overline{0}\right] \quad \& \quad \forall i>0\left[\left(c_{i}=\overline{0} \Rightarrow\right.\right. & \left.\left(c_{i-1} \in \bar{\Delta} \& c_{i+1}=\overline{0}\right)\right) \\
& \left.\&\left(c_{i} \in \sum-\{\overline{0}\} \Rightarrow c_{i-1} \in \Delta\right)\right] .
\end{aligned}
$$

Correct representation:

$$
\sum_{i=0}^{\infty} c_{i} 2^{i}=c
$$

To identify representations of 0 by just a small initial segment:

$$
\forall i \geqq 0\left[\left(c_{i+1}>0 \Rightarrow c_{i} \geqq 0\right) \&\left(c_{i+1}<0 \Rightarrow c_{i} \leqq 0\right)\right] .
$$

Under $(\mathrm{A})-(\mathrm{C}),(-2)^{i} 0 \overline{1} \overline{0}^{\infty}$ represents the integer 2 for all $i \geqq 0$. To prevent racing of the most significant nonzero digit to the first position, in just a few steps of the desired single head real-time simulator:

$$
\forall i \geqq 0\left[i \text { is odd } \Rightarrow\left|c_{i}\right|<2\right] .
$$


Now if $c_{0} c_{1} \cdots c_{m-1} c_{m} \cdots \in \operatorname{code}(c)$, with $c_{i} \in \Delta$ for $0 \leqq i \leqq m-1$, and $c_{i} \in \bar{\Delta}$ for $m \leqq i$, then for $m=0$ we have $0 \leqq|c| \leqq 2$, for $m=1$ we have $2 \leqq|c| \leqq 4$ and in general for $m \geqq 2$ :

$$
2^{m}-r \leqq|c| \leqq 2^{m+1}+r^{\prime}
$$

with

$$
r=2 \sum_{i=0, i \text { even }}^{m-2} 2^{i}+\sum_{i=1, i \text { odd }}^{m-2} 2^{i}, \quad r^{\prime}=2 \sum_{i=0, i \text { even }}^{m-1} 2^{i}+\sum_{i=1, i \text { odd }}^{m-1} 2^{i}
$$

which yields

$$
m-3<\log _{2}|c|<m+2 \text {. }
$$

Thus the length of the initial significant segment of the representation of $c \in \mathbb{Z}$ follows by and large the length of the usual binary representation of $|c|$. We are particularly interested in representations for zero. Note that the following proposition holds for code functions satisfying only $(\mathrm{A})-(\mathrm{C})$.

PROPOSITION 2. Let $c_{0} c_{1} \cdots c_{m} c_{m+1} \cdots \in \operatorname{code}(c)$ with $c \in \mathbb{Z}$. Then $c=0$ iff $c_{i}=\overline{0}$ for all $i \geqq 0$ iff $c_{0}=\overline{0}$.

Proof. By $(\mathrm{A}) c_{0}=\overline{0}$ iff $c_{i}=\overline{0}$ for all $i \geqq 0$. So we only have to prove $c=0$ iff $c_{0}=\overline{0}$. Assume $c_{i}=\overline{0}$ for all $i \geqq 0$. By (B) $c=0$. Assume $c_{i} \neq \overline{0}$ for some $i \geqq 0$. Then by (A) there exists a least $m \geqq i$ such that $c_{j}=\overline{0}$ for all $j>m$, and $\left|c_{m}\right| \neq 0$. For $m=0,|c| \geqq 1$, and for $m=1$ we have $|c| \geqq 2$. For $m \geqq 2$ :

$$
\begin{array}{rlrl}
|c| & =\left|\sum_{i=0}^{m} c_{i} 2^{i}\right| & & (\text { by }(\mathrm{B})) \\
& \geqq\left|2^{m}-\right| \sum_{i=0}^{m-1} c_{i} 2^{i}|| & \quad \text { (triangle inequality) } \\
& \geqq 2^{m}-2 \sum_{i=0}^{m-2} 2^{i} & (\text { by }(\mathrm{C})) \\
& =2 .
\end{array}
$$

2.2. Maintenance of the count. Let $S$ be the simulated quintessential counter and let $\delta_{1}, \delta_{2}, \cdots, \delta_{t}, \cdots, \delta_{i} \in\{-1,0,1\}$, be any fixed sequence of unit additions/subtractions. So at time $t \geqq 0, S$ contains the integer $\sum_{i=1}^{t} \delta_{i}$. We maintain the count in an array $C[0: \infty]$ such that the value of the array at time $t \geqq 0$ is $C^{t}[0: \infty] \in \operatorname{code}\left(\sum_{i=1}^{t} \delta_{i}\right)$, for any such input stream. The initial array $C^{0}[0: \infty]$ at time $t=0$ is defined by $C^{0}[i]=\overline{0}$ for all $i \geqq 0$, and therefore $C^{0}[0: \infty] \in \operatorname{code}(0)$. In the $t$ th simulated step, $t \geqq 1$, the current value $C^{t-1}$ of the array is mapped to the next value $C^{t}$ by a function COUNT $\left(t, \delta_{t}\right)$. The mapping COUNT is defined in terms of a composition of mappings, with the aid of an auxiliary function $I: \mathbb{N} \rightarrow 2^{\mathbb{N}}$, called the parameter selection function, which has as values sets of bounded cardinality (cardinality four suffices).

Definition. For $t \geqq 1$, let $I(t)=\left\{i_{l}, i_{l-1}, \cdots, i_{1}\right\}$ with $i_{l}>i_{l-1} \cdots>i_{1}$, and let $\delta \in\{-1,0,1\}$. COUNT $(t, \delta)$ is defined as a composition of mappings:

$$
\operatorname{COUNT}(t, \delta) \stackrel{\text { def }}{=} \operatorname{UPDATE}\left(i_{l}\right) ; \operatorname{UPDATE}\left(i_{l-1}\right) ; \cdots ; \operatorname{UPDATE}\left(i_{1}\right) ; \operatorname{INPUT}(\delta) .
$$

Hence

$$
C \stackrel{\operatorname{counT}(t, \delta)}{\rightleftharpoons} C^{\prime} \text {, with } C^{\prime} \neq \varnothing \text {, }
$$


if there exist $C_{l}, C_{l-1}, \cdots, C_{1} \neq \varnothing$ such that

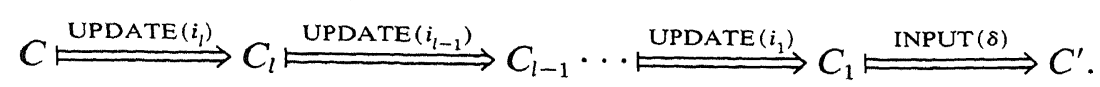

In all other cases $C \stackrel{\operatorname{COUNT}(t, \delta)}{\rightleftharpoons} \varnothing$.

Definition. Let $i \in \mathbb{N}$ and let $C[0: \infty]$ be a one-way infinite array, $C \neq \varnothing$.

$$
C[0: \infty] \stackrel{\operatorname{UPDATE}(i)}{\rightleftharpoons} C^{\prime}[0: \infty]
$$

$C^{\prime}[0: \infty] \neq \varnothing$, if UPDATE: $C[2 i: 2 i+3] \mapsto C^{\prime}[2 i: 2 i+3] \neq \varnothing$ and $C^{\prime}[0: 2 i-1]=$ $C[0: 2 i-1]$ and $C^{\prime}[2 i+4: \infty]=C[2 i+4: \infty]$, with the function UPDATE: $\Sigma^{4} \rightarrow \Sigma^{4}$ defined below. For convenience we first define UPDATE: $\Delta^{4} \rightarrow \Delta^{4}$ and then extend the mapping to $\Sigma^{4}$.

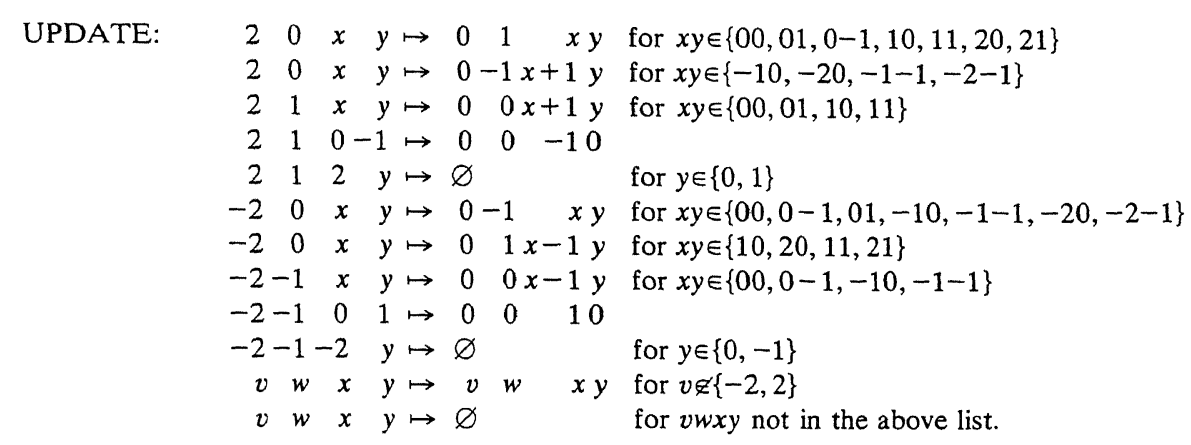

Extension of UPDATE to mappings from $\Sigma^{4}$ into $\Sigma^{4}$ : if $v w x y \notin \Delta^{4}$ then

$$
\text { UPDATE: } v w x y \mapsto v^{\prime} w^{\prime} x^{\prime} y^{\prime}
$$

for all $v w x y, v^{\prime} w^{\prime} x^{\prime} y^{\prime} \in \Delta^{*}(\bar{\Delta}-\{\overline{0}\})\{\overline{0}\}^{*} \cup\{\overline{0} \overline{0} \overline{0} \overline{0}\}$ such that the unbarred version of the mapping is in the previous list, and UPDATE: $v w x y \mapsto \varnothing$ in all other cases. (Recall that if $V$ is a finite alphabet, then $V^{*}$ is the set of all finite strings over $V$ including the empty string $\varepsilon$.)

Definition. Let $\delta \in\{-1,0,1\}$ and let $C[0: \infty]$ be a one-way infinite array, $C \neq \varnothing$.

$$
C[0: \infty] \stackrel{\text { INPUT }(\delta)}{=} C^{\prime}[0: \infty],
$$

$C^{\prime}[0: \infty] \neq \varnothing$, if $\operatorname{INPUT}_{\delta}: C[0: 1] \mapsto C^{\prime}[0: 1] \neq \varnothing$ and $C^{\prime}[2: \infty]=C[2: \infty]$ with INPUT $_{\delta}: \Sigma^{2} \rightarrow \Sigma^{2}$ defined below. For convenience we first define INPUT $\mathrm{IN}_{\delta}: \Delta^{2} \rightarrow \Delta^{2}$ and then extend the mapping to $\Sigma^{2}$.

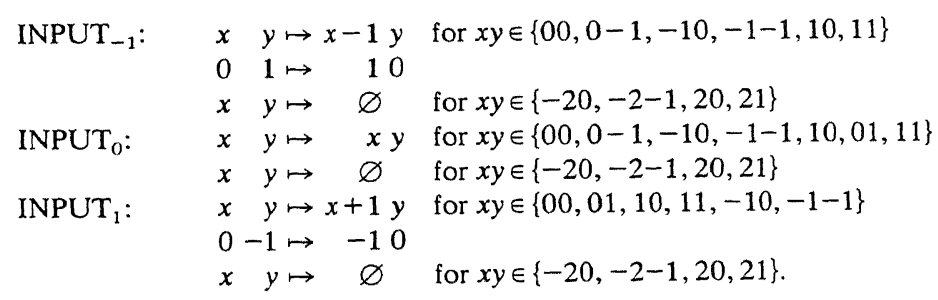

Extension of $\operatorname{INPUT}_{\delta}$ to mappings from $\Sigma^{2}$ into $\Sigma^{2}$ : if $x y \notin \Delta^{2}$ then

$$
\mathrm{INPUT}_{\delta}: x y \mapsto x^{\prime} y^{\prime}
$$

for all $x y, x^{\prime} y^{\prime} \in \Delta^{*}(\bar{\Delta}-\{\overline{0}\})\{\overline{0}\}^{*} \cup\{\overline{0} \overline{0}\}$ such that the unbarred version of the mapping is in the previous list and INPUT $\delta: x y \mapsto \varnothing$ in all other cases. 
If for some array $C[0: \infty]$ and $P=\operatorname{UPDATE}(i), i \geqq 0$, we have UPDATE: $C[2 i: 2 i+3] \mapsto \varnothing$ then $C \stackrel{P}{\Rightarrow} \varnothing$, by definition. If for some array $\underset{P}{C}[0: \infty]$ and $P=\operatorname{INPUT}(\delta), \delta \in\{-1,0,1\}$, we have $\operatorname{INPUT}_{\delta}: C[0: 1] \mapsto \varnothing$ then $C \stackrel{P}{\Leftrightarrow} \varnothing$, by definition. For all $P \in\{\operatorname{INPUT}(\delta)$, UPDATE $(i) \mid \delta \in\{-1,0,1\}, i \geqq 0\}$ we have by definition $\varnothing \stackrel{P}{\Rightarrow} \varnothing$, and thus $\stackrel{P}{\Rightarrow}$ is a mapping from $\Sigma^{\infty} \cup\{\varnothing\}$ into $\Sigma^{\infty} \cup\{\varnothing\}$ and not just a relation. Basically, INPUT $(\delta)$ adds the current input to the currently represented integer and UPDATE $(i)$ propagates carries and borrows in a segment of the representation, both preserving representations from the code function.

For each input sequence $D=\delta_{1}, \delta_{2}, \cdots, \delta_{t}, \cdots$, with $\delta_{t} \in\{-1,0,1\}$ for $t \geqq 1$, the sequence of mappings

$\operatorname{COUNT}(I, D) \stackrel{\text { def }}{=} \operatorname{COUNT}\left(1, \delta_{1}\right) ; \operatorname{COUNT}\left(2, \delta_{2}\right) ; \cdots ; \operatorname{COUNT}\left(t, \delta_{t}\right) ; \cdots$

defines a sequence of (a priori possibly undefined) arrays $C^{0}, C^{1}, \cdots, C^{t}, \cdots$ such that $C^{0}$ is the all-blank initial array $C^{0}[0: \infty]=\overline{0}^{\infty}$, and for all $t \geqq 1$ :

$$
C^{t-1} \stackrel{\operatorname{COUNT}\left(t, \delta_{t}\right)}{\rightleftharpoons} C^{t} \text {. }
$$

Decomposing COUNT $\left(t, \delta_{t}\right)$ into its constituent functions for all $t \geqq 1$, with $I(t)=$ $\left\{i_{t, l(t)}, i_{t, l(t)-1}, \cdots, i_{t, 1}\right\}$ and $i_{t, l(t)}>i_{t, l(t)-1}>\cdots>i_{t, 1}$, we obtain for each input sequence $D=\delta_{1}, \delta_{2}, \cdots, \delta_{t}, \cdots$ the sequence of basic mappings

$$
\begin{gathered}
\operatorname{COUNT}(I, D)=\operatorname{UPDATE}\left(i_{1, l(1)}\right) ; \operatorname{UPDATE}\left(i_{1, l(1)-1}\right) ; \cdots ; \operatorname{UPDATE}\left(i_{1,1}\right) ; \\
\operatorname{INPUT}\left(\delta_{1}\right) ; \operatorname{UPDATE}\left(i_{2, l(2)}\right) ; \cdots .
\end{gathered}
$$

In this sequence, the subsequence of mappings

$$
\begin{gathered}
\operatorname{COUNT}\left(t, \delta_{t}\right)=\operatorname{UPDATE}\left(i_{t, l(t)}\right) ; \operatorname{UPDATE}\left(i_{t, l(t)-1}\right) ; \cdots ; \operatorname{UPDATE}\left(i_{t, 1}\right) ; \\
\operatorname{INPUT}\left(\delta_{t}\right)
\end{gathered}
$$

is said to constitute the $t$ th step of the maintenance of array $C$. Starting from $C^{t-1}$ the sequence of intermediate arrays defined by the $t$ th step is

$$
C^{t-1}\left(=C_{t-1,0}\right), C_{t, l(t)}, C_{t, l(t)-1}, \cdots, C_{t, 1}, C_{t, 0}\left(=C^{t}\right)
$$

defined by

$$
C_{t-1,0} \stackrel{\operatorname{UPDaTE}\left(i_{t, l(t)}\right)}{\Longrightarrow} C_{t, l(t)} \cdots \stackrel{\operatorname{INPUT}\left(\delta_{t}\right)}{\rightleftharpoons} C_{t, 0}=C^{t} .
$$

Note that in the decomposition of $\operatorname{COUNT}(I, D)$ in the basic mappings $\operatorname{UPDATE}(\cdot)$ and INPUT $(\cdot)$ the parameter $t$ does not occur explicitly; the sequence of basic mappings is defined totally by the sequence of successive values of $I$ and the sequence of inputs. This is important in the next sections. In this section we show in Lemma 1 that, for any input sequence $D=\delta_{1}, \delta_{2}, \cdots$,

$$
C^{0}, C_{1, l(1)}, \cdots, C_{1,1} \in \operatorname{code}(0) \cup\{\varnothing\}
$$

and for all $t \geqq 1$

$$
C_{t-1,0}\left(=C^{t-1}\right), C_{t, l(t)}, \cdots, C_{t, 1} \in \operatorname{code}\left(\sum_{i=1}^{t-1} \delta_{i}\right) \cup\{\varnothing\} .
$$

In Proposition 3 it is demonstrated that for certain choices of the parameter selection function $I$ we have that $C_{t, j} \neq \varnothing$ for all $t \geqq 1$ and all $j, l(t) \geqq j \geqq 0$, whence $C^{t} \in$ code $\left(\sum_{i=1}^{t} \delta_{i}\right)$ for all $t \geqq 0$. 
Lemma 1. Let array $C \in \operatorname{code}(c)$ for some integer $c$. If, for some $i \geqq 0$, $C \stackrel{\text { UPDATE }(i)}{\Longrightarrow} C^{\prime}, C^{\prime} \neq \varnothing$, then $C^{\prime} \in \operatorname{code}(c)$. If, for some $\delta \in\{-1,0,1\}$, $C \stackrel{\text { INPUT }(\delta)}{\Longrightarrow} C^{\prime}, C^{\prime} \neq \varnothing$, then $C^{\prime} \in \operatorname{code}(c+\delta)$.

Proof. Let $C \in \operatorname{code}(c)$ for some integer $c$ and $C \stackrel{\text { UPDATE }(i)}{\Longrightarrow} C^{\prime}, C^{\prime} \neq \varnothing$, for some $i \geqq 0$. If $|C[2 i]| \neq 2$ then $C^{\prime}=C$ and there is nothing to prove. So let $|C[2 i]|=2$. Then, for $j<2 i$ and $j>2 i+3, C^{\prime}[j]=C[j]$. Since also $\sum_{j=0}^{3} C^{\prime}[2 i+j] 2^{2 i+j}=$ $\sum_{i=0}^{3} C[2 i+j] 2^{2 i+j}$, we have $\sum_{i=0}^{\infty} C^{\prime}[i] 2^{i}=\sum_{i=0}^{\infty} C[i] 2^{i}=c$. It is easy to check from the definition of UPDATE $(i)$, that if $(\mathrm{A}),(\mathrm{C})$ and $(\mathrm{D})$ hold for $C, C \stackrel{\operatorname{UPDATE}(i)}{\rightleftharpoons} C^{\prime}$ and $C^{\prime} \neq \varnothing$, then (A), (C) and (D) also hold for $C^{\prime}$. Hence $C^{\prime} \in \operatorname{code}(c)$. Let $C \in \operatorname{code}(c)$ for some integer $c$ and $C \stackrel{\text { INPUT }(\delta)}{\Longrightarrow} C^{\prime}, C^{\prime} \neq \varnothing$, for some $\delta \in\{-1,0,1\}$. Since $C^{\prime} \neq \varnothing$ we have $|C[0]|<2$. For all $j>1, C^{\prime}[j]=C[j]$. Because also $C^{\prime}[0]+2 C^{\prime}[1]=$ $C[0]+2 C[1]+\delta$ we have $\sum_{i=0}^{\infty} C^{\prime}[i] 2^{i}=c+\delta$. It is easy to check from the definition of INPUT ( $\delta$ ) that if (A), (C) and (D) hold for $C, C \stackrel{\operatorname{INPUT}(\delta)}{\rightleftharpoons} C^{\prime}$, and $C^{\prime} \neq \varnothing$, then (A), (C) and (D) also hold for $C^{\prime}$. Hence $C^{\prime} \in$ code $(c+\delta)$.

Proposition 3. Let $T: \mathbb{N} \rightarrow \mathbb{N}$ be any function such that $T(i) \leqq 3^{i}$ for all $i \geqq 0$. Let the parameter selection function $I: \mathbb{N} \rightarrow 2^{\mathbb{N}}$, associated with the mapping COUNT, be such that for all indices $i \geqq 0$ and steps $t \geqq 1$ there exists $a t^{\prime}, t \leqq t^{\prime}<t+T(i)$ and $i \in I\left(t^{\prime}\right)$. Then for each input sequence $\delta_{1}, \delta_{2}, \cdots, \delta_{t}, \cdots, \delta_{t} \in\{-1,0,1\}, t \geqq 1$, there exists a sequence of one-way infinite arrays $C^{0}, C^{1}, \cdots, C^{t}, \cdots$, with $C^{0}$ the all blank initial array and $C^{t-1}$ is mapped to $C^{t}$ by $\operatorname{COUNT}\left(t, \delta_{t}\right)$ for all $t \geqq 1$, such that $C^{t} \in$ code $\left(\sum_{i=1}^{t} \delta_{i}\right)$ for all $t \geqq 0$.

Proof. Roughly speaking the proposition states that if, starting from the all blank initial array $C^{0}$, UPDATE $(i)$ is executed at least once in every interval of $3^{i}$ steps, for all $i \geqq 0$, and INPUT $(\delta)$ is executed each step, with $\delta \in\{-1,0,1\}$ the currently polled input, then the array at time $t$ represents the stored integer at time $t$ according to the code function. By Lemma 1 and the definition of COUNT this is the case if, under the timing assumption on the parameter selection function $I$, each time UPDATE $(i)$ and INPUT $(\delta)$ map an array satisfying (A), (C) and (D), the result is not the undefined array $\varnothing$. The only way UPDATE $(i)$ can map an array $C[0: \infty]$, satisfying (A), (C) and (D) to $\varnothing$ is for $C[2 i: 2 i+2] \in\{212,21 \overline{2},-2-1-2,-2-1-\overline{2}\}$. Similarly, the only way INPUT $(\delta)$ can map an array $C[0: \infty]$ satisfying $(\mathrm{A}),(\mathrm{C})$ and (D) to $\varnothing$ is for $C[0] \in\{2, \overline{2},-2,-\overline{2}\}$. Hence we have to prove that, under the assumptions on $I$, and starting from the all blank initial array $C^{0}$, these undesirable subarrays do not occur at the crucial moments. Induction is on the number of steps $t$.

Base case: the first step. Since $C^{0}$ is all-blank, for all $i \geqq 0$ we have $C^{0}[2 i: 2 i+3]=$ $\overline{0} \overline{0} \overline{0}$. Hence $C^{0} \stackrel{\operatorname{COUNT}\left(1, \delta_{1}\right)}{\rightleftharpoons} C^{1}$ with $C^{1}[0]=\bar{\delta}_{1}$ and $C^{1}[i]=\overline{0}$ for all $i \geqq 1$. That is, $C^{1} \in \operatorname{code}\left(\delta_{1}\right)$.

Induction: $t \geqq 1$. Assume, by way of contradiction, that for the input sequence $\delta_{1}, \delta_{2}, \cdots, \delta_{t}\left(\delta_{j} \in\{-1,0,1\}, 1 \leqq j \leqq t\right)$ we have for all $j, 1 \leqq j \leqq t$ :

$$
C^{j-1} \stackrel{\operatorname{CounT}\left(j, \delta_{j}\right)}{\rightleftharpoons} C^{j}, C^{j} \neq \varnothing \quad \text { (induction assumption), }
$$

and

$$
C^{t} \stackrel{\operatorname{COUNT}(t+1, \delta)}{\Longrightarrow} \varnothing \quad \text { (contradictory assumption), }
$$

for some $\delta \in\{-1,0,1\}$. For all $j, 1 \leqq j \leqq t$, by Lemma $1, C^{j} \in \operatorname{code}\left(\sum_{i=1}^{j} \delta_{i}\right)$. Let $I(t+$ $1)=\left\{i_{1}, i_{l-1}, \cdots, i_{1}\right\}$ and $i_{l}>i_{l-1}>\cdots>i_{1}$. Decomposing COUNT $(t+1, \delta)$ into its 
constituent mappings we have

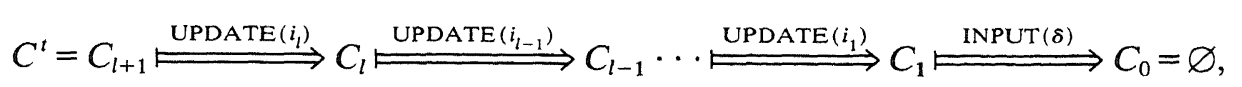

for some intermediate, possibly undefined, arrays $C_{l}, C_{l-1}, \cdots, C_{0}$. By the contradictory assumption there must be a first undefined array in this sequence, say $C_{j-1}=\varnothing$ and $C_{j} \neq \varnothing$ for some $j, 0<j \leqq l+1$. Note that, by Lemma $1, C_{j} \in \operatorname{code}\left(\sum_{i=1}^{t} \delta_{i}\right)$.

Case 1. $j>1$. Setting $i$ to $i_{j-1}$, to avoid subscripts,

$$
C_{j} \stackrel{\operatorname{UPDATE}(i)}{\rightleftharpoons} \varnothing \text {. }
$$

Since $C_{j} \in \operatorname{code}\left(\sum_{i=1}^{t} \delta_{i}\right)$ and therefore satisfies (A), (C) and (D), this can happen only if $C_{j}[2 i: 2 i+2] \in\{-2-1-2,-2-1-\overline{2}, 212,21 \overline{2}\}$. Assume $C_{j}[2 i: 2 i+2]=212$, the other cases being symmetrical. Since the initial array $C^{0}$ contained only blanks, there must be a $t^{\prime}, 0<t^{\prime} \leqq t$, with $t-t^{\prime}$ minimal, such that

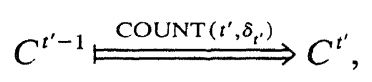

$C^{t^{\prime}}[2 i+2]=2$ and $C^{t^{\prime}-1}[2 i+2] \neq 2$. (A previous mapping UPDATE $(k)$, with $k>i$, in the $(t+1)$ th step could not have set $C[2 i+2]$ to 2 from another value, so if $C_{j}[2 i+2]=2$ then $C_{k}[2 i+2]=2$ for all $k, l+1 \geqq k \geqq j$. Since $C_{l+1}=C^{t}$ indeed $t^{\prime} \leqq t$.) From the definitions it follows that $C[2 i+2]$ can be set to 2 , from another value, only by the mapping UPDATE $(i)$. So $i \in I\left(t^{\prime}\right)$, and we denote by $C^{\prime}$ the array mapped upon by the occurrence of $\operatorname{UPDATE}(i)$ in $\operatorname{COUNT}\left(t^{\prime}, \delta_{t^{\prime}}\right)=\operatorname{UPDATE}\left(i_{t^{\prime}}^{\prime}\right)$; $\operatorname{UPDATE}\left(i_{l^{\prime}-1}^{\prime}\right) ; \cdots ; \operatorname{UPDATE}\left(i_{1}^{\prime}\right) ; \operatorname{INPUT}\left(\delta_{t^{\prime}}\right)$. By the definition of UPDATE $(i)$ we must have $C^{\prime}[2 i: 2 i+2]=002$. Since during the mappings, following UPDATE $(i)$ in COUNT $\left(t^{\prime}, \delta_{t^{\prime}}\right)$, subarray $C[2 i+2: \infty]$, is not accessed, and we have by Lemma 1 that $C^{\prime} \in \operatorname{code}\left(\sum_{i=1}^{t^{-1}} \delta_{i}\right)$ and $C^{t^{\prime}} \in \operatorname{code}\left(\sum_{i=1}^{t^{\prime}} \delta_{i}\right)$, it therefore follows that

$$
\sum_{k=0}^{2 i+1} C^{t^{\prime}}[k] 2^{k}=\sum_{k=0}^{2 i+1} C^{\prime}[k] 2^{k}+\delta_{t^{\prime}}=\sum_{k=0}^{2 i-1} C^{\prime}[k] 2^{k}+\delta_{t^{\prime}} \leqq\left(4^{i+1}-1\right) / 3 \quad \text { (by (C) and (D)). }
$$

Any first occurrence of an UPDATE $(i+1)$ in a COUNT $\left(t^{\prime \prime}, \delta_{t^{\prime \prime}}\right), t^{\prime}<t^{\prime \prime}<t+1$, so in between the mappings by the two occurrences of UPDATE $(i)$ in steps $t^{\prime}$ and $t+1$, would have set $C[2 i+2]$ to 0 , resulting in $\left|C^{t^{\prime \prime}}[2 i+2]\right| \leqq 1$, contradicting the minimality of $t-t^{\prime}$. Therefore, for all $t^{\prime \prime}, t^{\prime}<t^{\prime \prime}<t+1, i+1 \notin I\left(t^{\prime \prime}\right)$. By the assumption on $I$ in the proposition it follows that

$$
t-t^{\prime}<3^{i+1}
$$

We are now ready to derive a contradiction. For the only mappings which can alter something in $C[2 i+2: 2 i+3]$ are UPDATE $(i)$ and UPDATE $(i+1)$. However, in between the mappings according to the occurrence of UPDATE $(i)$ in step $t^{\prime}$ and that of UPDATE $(i)$ in step $t+1$, no occurrence of UPDATE $(i)$ has changed $C[2 i+2: 2 i+$ $3]$ (since this would contradict the minimality of $\left.t-t^{\prime}\right)$, and UPDATE $(i+1)$ has not occurred at all (since $C_{j}[2 i+2] \neq 0$ by assumption, $i+1$ is not in $I(t+1)$ too). So, by the definitions of COUNT and UPDATE we obtain:

$$
\sum_{k=2 i+2}^{\infty} C^{t^{\prime}}[k] 2^{k}=\sum_{k=2 i+2}^{\infty} C_{j}[k] 2^{k} .
$$


Furthermore, by Lemma 1,

$$
\sum_{k=0}^{\infty} C^{t}[k] 2^{k}=\sum_{k=1}^{t} \delta_{k}=\sum_{k=0}^{\infty} C_{j}[k] 2^{k}
$$

Thus:

$$
\begin{aligned}
\sum_{k=0}^{2 i+1} C_{j}[k] 2^{k} & =\sum_{k=0}^{\infty} C_{j}[k] 2^{k}-\sum_{k=2 i+2}^{\infty} C_{j}[k] 2^{k} \\
& =\sum_{k=1}^{t} \delta_{k}-\sum_{k=2 i+2}^{\infty} C_{j}[k] 2^{k} \\
& =\sum_{k=1}^{t} \delta_{k}-\sum_{k=2 i+2}^{\infty} C^{t^{\prime}}[k] 2^{k} \\
& =\sum_{k=1}^{t} \delta_{k}-\sum_{k=1}^{t^{\prime}} \delta_{k}+\sum_{k=0}^{2 i+1} C^{t^{\prime}}[k] 2^{k} \\
& \leqq t-t^{\prime}+\left(4^{i+1}-1\right) / 3 \\
& <3^{i+1}+\left(4^{i+1}-1\right) / 3 .
\end{aligned}
$$

But, by way of contradiction, it was assumed that $C_{j}[2 i: 2 i+1]=21$. Therefore,

$$
\sum_{k=0}^{2 i+1} C_{j}[k] 2^{k}=4^{i+1}+\sum_{k=0}^{2 i-1} C_{j}[k] 2^{k} \geqq 4^{i+1}-\left(4^{i}-4\right) / 3-4^{i} / 2,
$$

for $i \geqq 2$ (and $\geqq 14$ for $i=1$, $\geqq 4$ for $i=0$ ), by (C) and (D). Since for all $i \geqq 0$ the contradictory assumption leads to the contradictory inequalities (5) and (6) we conclude that $j=1$ and case 2 holds.

Case 2. $j=1$ and

$$
C_{1} \stackrel{\operatorname{InpuT}(\delta)}{\Longrightarrow} \varnothing \text {. }
$$

However, under the assumptions in the Proposition, $0 \in I(t)$ for all $t \geqq 1$, so COUNT ( $t+$ $1, \delta)=\cdots$; UPDATE $(0)$; INPUT $(\delta)$. But if $C_{1}[0: \infty] \neq \varnothing$ is the value of UPDATE (0) then $C_{1}[0] \notin\{-2,-\overline{2}, 2, \overline{2}\}$. Therefore, the contradictory assumption also fails in this case and

$$
C_{1} \stackrel{\operatorname{INPUT}(\delta)}{\rightleftharpoons} C_{0} \neq \varnothing .
$$

Since the contradictory assumption has now been proven false, by Lemma 1 $C_{l+1}, C_{l}, \cdots, C_{1} \in \operatorname{code}\left(\sum_{i=1}^{t} \delta_{i}\right)$ and $C_{0} \in \operatorname{code}\left(\sum_{i=1}^{t} \delta_{i}+\delta\right)$. Setting $C^{t+1}=C_{0}$ completes the induction.

Proposition 3 shows us a way of real-time simulating the quintessential counter $S$ figuring in Proposition 1. Let $C^{0}$ be the all-blank initial array, and let the parameter selection function $I$ meet the timing conditions in Proposition 3. If we map in the $t$ th step, for each $t \geqq 1$, the current array value to the next one by COUNT $(t, \delta)$, where "add $\delta ", \delta \in\{-1,0,1\}$, is the input command polled from the input terminal in the $t$ th step, then the array at each time $t \geqq 0$ is a representation from code (stored integer at time $t$ ). Since the mapping $\operatorname{COUNT}(t, \delta)=\cdots ; \operatorname{INPUT}(\delta)$, and $\operatorname{INPUT}(\delta)$ maps $C[0: 1]$ to a next value, we can simultaneously output "count equals zero" if the next value of $C[0]=\overline{0}$, or "count unequal zero" if the next value of $C[0] \neq \overline{0}$, according to Proposition 2. 
Note that the requirement of an initial zero count is not essential. We can as well prove Proposition 3 starting from $C^{0}$ equals a representation of an arbitrary integer $c$. For instance, a representation from code $(c)$, containing only equal signed digits of absolute value less than 2 , for $C^{0}$, lets Proposition 3 go through as well. Thus, the arrangement can real-time simulate initially nonzero counters.

2.3. An oblivious one-head tape unit. Proposition 3 puts a heavy burden on a one-head tape unit: $C[0: 3]$ must always be under scan, $C[2: 5]$ within each third step, and in general $C[2 i: 2 i+3]$ at least once within each interval of $3^{i}$ steps, for all $i \geqq 0$ simultaneously. This requires that, basically, at all times all $C[i]$ must be on the move, drifting inward or outward from the location occupied by the single head, so to speak. This data motion must be due to the swapping of array elements amongst the momentarily simultaneously scanned tape squares. To be able to scan $C[2 i: 2 i+3]$ within certain time intervals, for all $i \geqq 0$, it is necessary that at certain times arbitrarily many of such quadruples are split and the pieces geometrically far apart. The piece $C[2 i: 2 i+$ 1] must be joined to piece $C[2 i-2: 2 i-1]$ at certain times and to $C[2 i+2: 2 i+3]$ at other times, for all $i \geqq 1$. Apart from performing the splitting, moving and glueing, the head must also recognize quadruples $C[2 i: 2 i+3]$ to perform UPDATE, and also know the relative order amongst pairs of such foursomes. Hence we need to maintain some order and identification of the array elements. Yet we cannot identify the individual elements of $C$ with respect to their position, since such an identification tag for $C[i]$ needs $\log i$ space and $\log i$ time to evaluate. All this points in the direction of a recursive process, but again we cannot maintain depth of recursion parameters.

The process exhibited below rests on the following intuition. The goal is roughly to access quadruples of consecutive elements of $C$, of index $\Theta(i)$, at least once in each interval of $2^{\Theta(i)}$ steps, for all $i \geqq 0$. We call the individual array elements cells and consider them as packets of information to be swapped amongst simultaneously scanned squares. Assume we are able to move a block of cells, called $A_{1}$, by, according to some regime, moving the head, centered on the cells constituting $A_{1}$, from the left end of $A_{1}$, where it scans some squares left adjacent to $A_{1}$, to the right end of $A_{1}$, where it scans some squares right adjacent to $A_{1}$, and back again to the left end of $A_{1}$. Let $A_{1}$ be contained in a block of cells called $A_{2}$. Then $A_{1}$ moves by transporting cells of $A_{2}-A_{1}$ through $A_{1}$ to the other side of $A_{1}$, while simultaneously shifting the cells of $A_{1}$. Thus, we will shift the total block $A_{1}$ from the left end of $A_{2}$ to the right end, and back again to the left end. During such a full sweep of $A_{1} \operatorname{over} A_{2}$, we will shift block $A_{2}$ within a larger block $A_{3}$ by a single square. So the relation between $A_{2}$ and $A_{3}$ is analogous to that between $A_{1}$ and $A_{2}$. See Fig. 1 .

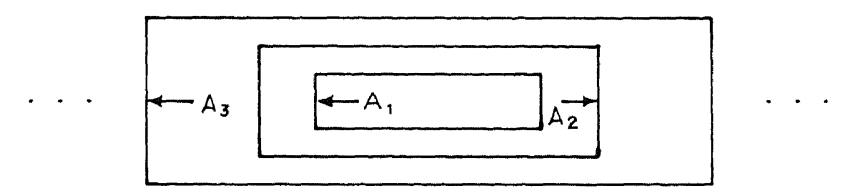

FIG. 1. The blocks are individually "moving" in the indicated directions.

In general, we envision an infinite series of nested blocks, $A_{1}, A_{2}, \cdots, A_{i}, A_{i+1}, \cdots$, with $A_{i}$ properly embedded in $A_{i+1}, i \geqq 1$, such that a full sweep of block $A_{i}$ over block $A_{i+1}$ shifts block $A_{i+1}$ one square in the currently desired direction. In the above arrangement, the head is always centered on block $A_{1}$, and therefore, since it is allowed to scan but a fixed number of squares, when it is centered 
at the end of block $A_{1}$ it scans but a fixed amount of squares outside. Since the ends of the individual blocks govern the action the single head ought to take, and also cells of $A_{i+1}-A_{i}$ have to be transported through $A_{i}$ for arbitrary $i$, we cannot have the physically present ends of all of blocks $A_{2}, A_{3}, \cdots, A_{i}$ in between the head centered on $A_{1}$ and a cell, to be transported, in $A_{i+1}-A_{i}$. So we want the blocks to move, in a sense, completely out of each other. That is, an arrangement as below in Fig. 2 , where we denote the cells in $A_{i+1}-A_{i}$ as $B_{i+1}$, for all $i \geqq 1$, and $A_{1}$ by $B_{1} .(x \stackrel{*}{\Rightarrow} y$ denotes that $y$ occurs after $x$.)

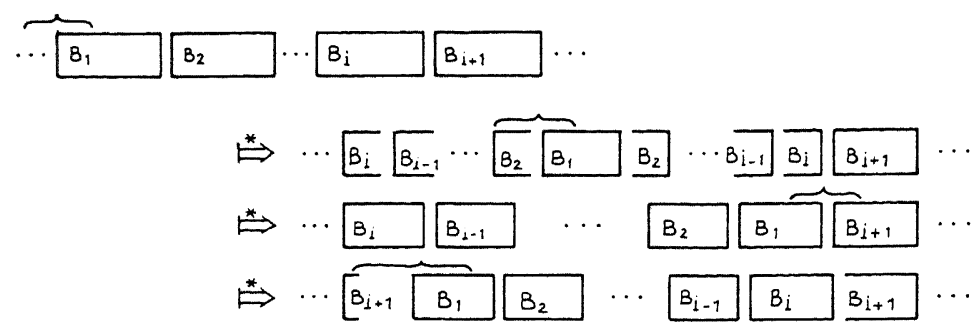

FIG. 2
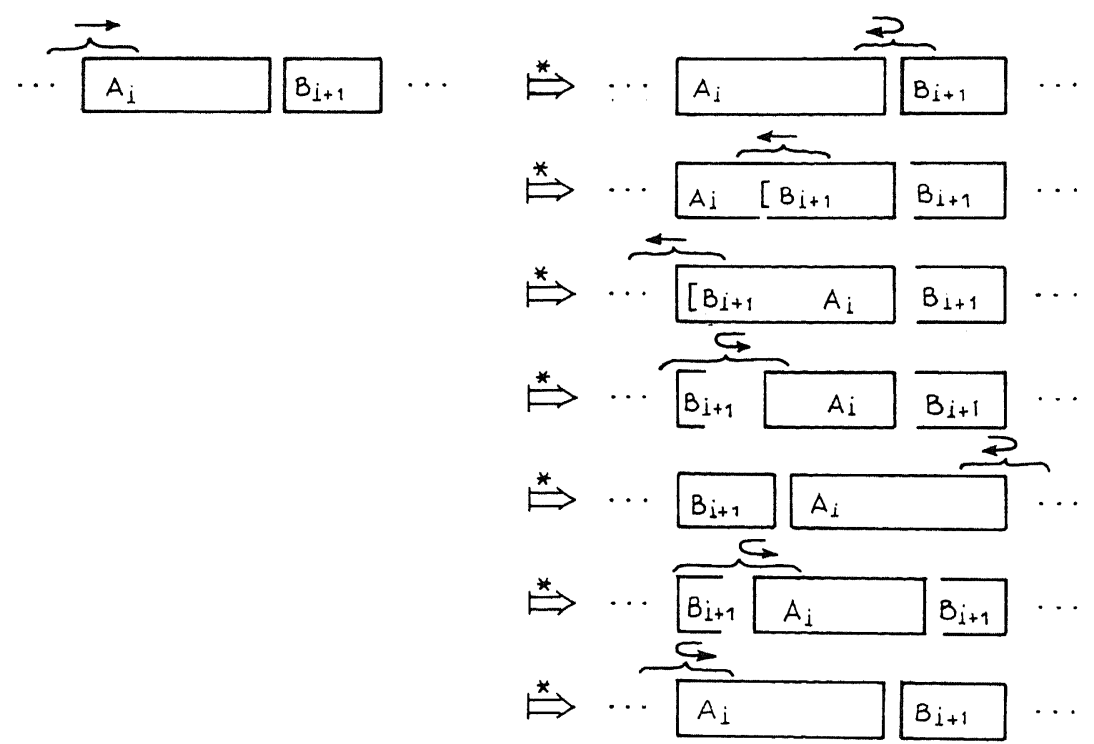

FIG. 3. The action of block $A_{i+1}=A_{i} \cup B_{i+1}$ with respect to blocks $B_{j}, j>i+1$ is not depicted.

In this manner we telescope the blocks, as it were, inwards and then outwards in the other direction, subsequently reversing the process. To achieve this behavior, we transport, for all $i \geqq 1$, elements of block $B_{i+1}$ through $A_{i}=\bigcup_{j=1}^{i} B_{j}$ while simultaneously shifting the cells of $A_{i}$ to accommodate the transport. The motion of the head through $A_{i}$ is governed by recursively moving $B_{j+1}$ through $A_{j}$, for all $j, j \leqq i$. Schematically, level $i$ of the process is depicted in Fig. 3. When the head was at the ends of block $A_{i+1}=A_{i} \cup B_{i+1}$, it now could have picked up or deposited a cell outside, that is, of a block $B_{j}, j>i+1$. Assume that all blocks $B_{i}, i \geqq 1$, have the same number of cells, say $x$. By a full sweep of the head over block $A_{i}$ we shall mean the action the head has to perform, starting from one end of $A_{i}$, to pick up a cell of $B_{j}, j>i$, deposit 
it on the other side of $A_{i}$, and finish at the same end of $A_{i}$ from which it started. So basically a full sweep of the head is a traversal of block $A_{i}$ from one end to the other end and back again. Let a full sweep of the head over $A_{i}$ take at most $S(i)$ steps, $i \geqq 1$. Then to transport all of $B_{i+1}$ from one end of $A_{i}$ to the other end, and back again, takes at most $c x S(i)$ steps for some constant $c$. Since this constitutes a full sweep of the head over block $A_{i+1}$, we have $S(i+1) \leqq c x S(i)$, for all $i \geqq 1$, and obviously $S(1) \leqq c x$. So $S(i) \in 2^{O(i)}$.

In the formal construction below we set the block size to 2 , and represent the loosely described block $B_{i}$ by " $\left[{ }_{i}\right]_{i}$ ", in the understanding that the two cells concerned are tagged with "[" and "]". The subscripts on the tags are just there to aid the reader, but do not occur in the actual simulation. An element of block $B_{j}$ in transport through block $A_{i}, j>i$, is identified by a curly bracket of the appropriate type. Thus each individual cell has permanently assigned to it a tag, consisting of either an opening or closing bracket, which may at different times be square or curly. Fig. 4 sketches a descriptive situation:

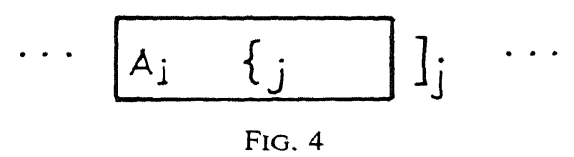

After these preliminaries we formally define a one-head tape unit $M$. It is convenient to view the instantaneous descriptions (i.d.'s) (momentary snapshots of $M$ 's tape contents and the head position) as one-way infinite linear arrays $T$, with " $<$ " or " $>$ " denoting the center of the head position. We tag the cells, containing elements of the array $C$ of the previous section, with "[", "]", "\{" or " $\} "$ ". Below we display only these tags, since for the moment we are not interested in the cell contents. The identity of the underlying squares is not important, but the identity of the tagged cells is fixed, wherever they end up. For convenience of the reader we index the tagged cells (or rather the tags). The eventually defined machine, however, has no indexes associated with the cells, only one out of the four mentioned tags. The initial i.d. is, now focussing on the tags only,

$$
T^{0}=>\left[_{1}\right]_{1}\left[_{2}\right]_{2} \cdots\left[_{i}\right]_{i}\left[{ }_{i+1}\right]_{i+1} \cdots
$$

We describe transformations of the array $T$ in the form of six parametrized recursive functions, and four nonparametrized functions, each of two types. Each such function $X$ will, for a unique subarray of $T$, rewrite this subarray by reordering its elements, specified by $X(\cdot): \alpha \diamond \beta \mapsto \alpha^{\prime} \diamond^{\prime} \beta^{\prime}$ with $\alpha, \alpha^{\prime}, \beta, \beta^{\prime}$ being strings of (for clarity indexed) tags and $\diamond, \diamond^{\prime} \in\{>,<\}$. A definite requirement for the process is that, at some time, it has to scan " $\left[{ }_{i+2}\right]_{i+2}\left[{ }_{i+3}\right]_{i+3}$ " for the first time. So we define, for all $i \geqq 0$ :

$$
\left.A(>, i):>\left[_{1}\right]_{1}[]_{2}\right]_{2} \cdots\left[\left[_{i}\right]_{i}\left[_{i+1}\right]_{i+1} \mapsto\left[_{i+1}\left[_{i}\right]_{i}\left[_{i-1}\right]_{i-1} \cdots[]_{1}\right]_{1}>\right]_{i+1} .
$$

For symmetry we also define:

$$
\left.A(<, i):\left[{ }_{i+1}\right]_{i+1}\left[{ }_{i}\right]_{i}\left[{ }_{i-1}\right]_{i-1} \cdots[]_{1}\right]_{1}<\mapsto\left[{ }_{i+1}<\left[_{1}\right]_{1}\left[_{2}\right]_{2} \cdots\left[{ }_{i}\right]_{i}\right]_{i+1} .
$$

To abbreviate notation we shall henceforth denote shortly, for all $i \geqq 0$,

$$
\begin{gathered}
\diamond\left[_{I}\right]_{I} \stackrel{\text { def }}{=} \diamond\left[[ _ { 1 } ] _ { 1 } [ _ { 2 } ] _ { 2 } \cdots \left[\left[_{i}\right]_{i},\right.\right. \\
\left.[]_{I} \diamond \stackrel{\text { def }}{=}[]_{i}\right]_{i}\left[_{i-1}\right]_{i-1} \cdots\left[\left[_{1}\right]_{1} \diamond,\right.
\end{gathered}
$$


with

So for all $i \geqq 0$ :

$$
\diamond \in\{<,>\} \text {, and }\left[{ }_{I}\right]_{I}=\varepsilon \text { for } i=0 .
$$

$$
\begin{aligned}
& A(>, i):>\left[_{I}\right]_{I}\left[_{i+1}\right]_{i+1} \mapsto\left[_{i+1}[I]_{I}>\right]_{i+1}, \\
& \left.\left.A(<, i):\left[_{i+1}\right]_{i+1}[]_{I}\right]_{I}<\mapsto\left[_{i+1}<[]_{I}\right]_{I}\right]_{i+1} .
\end{aligned}
$$

By execution of $A(>, i)$ on the appropriate unique substring of $T^{0}$ we have therefore, using the same rewriting denotation as in the previous section:

with

$$
T^{0} \stackrel{A(>, i)}{\rightleftharpoons} T^{t_{i}}
$$

and

$$
T^{0}=>\left[{ }_{1}\right]_{1}[2]_{2} \cdots\left[[ _ { i } ] _ { i } \left[\left[_{i+1}\right]_{i+1}\left[_{i+2}\right]_{i+2}[i+3]_{i+3} \cdots\right.\right.
$$

$$
T^{t_{i}}=\left[_{i+1}\left[_{i}\right]_{i}\left[_{i-1}\right]_{i-1} \cdots\left[_{1}\right]_{1}>\right]_{i+1}\left[{ }_{i+2}\right]_{i+2}\left[{ }_{i+3}\right]_{i+3} \cdots
$$

where $t_{i}$ is the number of steps it takes to execute the mapping $A(>, i)$, to be specified later, $i \geqq 0$.

With the head scanning at least five squares right of the center position, indicated by " $>$ ", the subarray " $\left[{ }_{i+2}\right]_{i+2}\left[_{i+3}\right]_{i+3}$ " is scanned at time $t_{i}$, for all $i \geqq 0$, while at time $t=0$ the subarray " $\left.\left[{ }_{1}\right]_{1}[]_{2}\right]_{2}$ " is scanned.

Definition. To achieve the required interchange of tagged cells, define the functions below. Recall that " $\}_{j}$ " denotes the same cell as " $]_{j}$ ", only the attached tags have changed. Similarly for " $\ell_{j}$ " and " $\left[L_{j}\right.$ ". For all $i \geqq 0$ and $j>i+1$ :

$$
\begin{aligned}
& A(>, i):>\left[{ }_{I}\right]_{I}\left[_ { i + 1 } x \mapsto \left[_{i+1}\left[_{I}\right]_{I}>x \quad(x \neq[)\right.\right. \\
& \left.A(<, i): x]_{i+1}\left[{ }_{I}\right]_{I}<\mapsto x<\left[\left[_{I}\right]_{I}\right]_{i+1} \quad(x \neq]\right) \\
& \left.\left.\left.\left.B(>, i):]_{i+1}[]_{I}\right]_{I}>\right]_{j} x \mapsto\right\}_{j}<\left[_{I}\right]_{I}\right]_{i+1} x \quad(x \neq[) \\
& B(<, i): x \mathrm{~L}_{j}<\left[_{I}\right]_{I}\left[_ { i + 1 } \mapsto x \left[_{i+1}\left[{ }_{I}\right]_{I}>\mathcal{L}_{j} \quad(x \neq \mathrm{]})\right.\right. \\
& \left.\left.C(>, i):]_{i+1}[]_{I}\right]_{I}>\right]_{j}\left[_{j+1} \mapsto\right\}_{j}\left\{_{j+1}<\left[_{I}\right]_{I}\right]_{i+1} \\
& C(<, i):]_{j+1}\left[_{j}<\left[_{I}\right]_{I}\left[_ { i + 1 } \mapsto [ _ { i + 1 } [ I _ { I } ] _ { I } > \} _ { j + 1 } \left\{_{j}\right.\right.\right. \\
& D(>, i):>\left[_{I}\right]_{I} x \mapsto\left[_{I}\right]_{I}>x \quad(x \neq[) \\
& D(<, i): x\left[\left[_{I}\right]_{I}<\mapsto x<\left[_{I}\right]_{I} \quad(x \neq \mathbf{]})\right. \\
& \left.E(>, i):\left[_{i+1}[]_{I}\right]_{I}>\right]_{i+1} x \mapsto\left[_{i+1}\right]_{i+1}\left[_{I}\right]_{I}>x \quad(x \neq \mathbb{D}) \\
& \left.E(<, i): x\left[\left[_{i+1}<[]_{I}\right]_{I}\right]_{i+1} \mapsto x<[]_{I}\right]_{I}\left[_{i+1}\right]_{i+1} \quad(x \neq \mathbf{]}) \\
& \left.F(>, i):\left[{ }_{i+1}[]_{I}\right]_{I}>\right]_{i+1}\left[{ }_{i+2} x \mapsto \coprod_{i+2}\left[{ }_{i+1}\right]_{i+1} \coprod_{I}\right]_{I}>x \quad(x \neq[)
\end{aligned}
$$

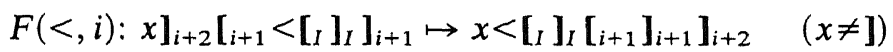

$$
\begin{aligned}
& G(>):>\left\{_{j}\right]_{i}\left[_{i+1} \mapsto>\right]_{i}\left[_ { i + 1 } \left\{_{j}\right.\right. \\
& \left.\left.G(<):]_{i+1}\left[{ }_{i}\right\}_{j}<\mapsto\right\}_{j}\right]_{i+1}\left[_{i}<\right. \\
& H(>):>\}_{j+1}\left\{_{j}\right]_{i}\left[_{i+1} \mapsto>\right]_{i}\left[_{i+1}\right\}_{j+1}\left\{_{j}\right. \\
& H(<):]_{i+1}\left[_{i}\right\}_{j}\left\{_{j+1}<\mapsto\right\}_{j}\left\{_{j+1}\right]_{i+1}\left[_{i}<\right. \\
& \left.\left.J(>):>\left\{{ }_{i+1}\right]_{i}\right]_{i+1} \mapsto<\right]_{i}\left[{ }_{i+1}\right]_{i+1} \\
& J(<):\left[{ }_{i+1}\left[{ }_{i}\right\}_{i+1}<\mapsto\left[{ }_{i+1}\right]_{i+1}\left[{ }_{i}>\right.\right.
\end{aligned}
$$




$$
\begin{aligned}
& \left.\left.K(>):>\}_{i+2}\left\{\left\{_{i+1}\right]_{i}\right]_{i+1} \mapsto<\right]_{i}\left[_{i+1}\right]_{i+1}\right]_{i+2} \\
& K(<):\left[_ { i + 1 } [ _ { i } \} _ { i + 1 } \left\{_{i+2}<\mapsto\left[{ } _ { i + 2 } [ { } _ { i + 1 } ] _ { i + 1 } \left[_{i}>\right.\right.\right.\right.
\end{aligned}
$$

The parametrized functions $A$ through $F$ set the basic pattern to transport tagged cells from one side of $\left[_{I}\right]_{I}$ to the other side. (The index $j$ is always greater than $i+1$.) The nonparametrized functions $G$ and $H$ serve to move (linked pairs of) curly brackets through "][" interfaces. If curly brackets are adjacent to a (linked) pair like "][" then they have not yet reached their destination. If curly brackets are adjacent to a pair of square brackets of equal type, then they have reached their destination, and are fitted in place and changed back to square brackets, by the functions $J$ and $K$. In the $G$ and $H$ functions, the index $j$ is again greater than $i+1$. However, to make the point once more, the indexes are only put there to aid the reader. The intention of the described rewritings is that the arrays concerned consist of nonsubscripted brackets, each bracket viewed as tagging a particular cell. The rewriting reorders these tagged cells in the array, and possibly changes brackets from square ones to curly ones of the same type, or vice versa, as indicated in the indexed version above. Note that $A(>, i): Y \mapsto Y^{\prime}$ and $A(<, i): Z \mapsto Z^{\prime}$ are related by the fact that $Z$ is the mirror image of $Y$ and $Z^{\prime}$ is the mirror image of $Y^{\prime}$. With mirror image we do not mean only the reverse, but the reverse with every constituent symbol changed to its mirror image, so " $>$ " to "<", "[" to "]", "[" to "]", " $\{$ " to " \}" and "\}" to " $\{$ ". Similarly for the other functions.

LEMMA 2. For all $i>0$, the functions are related as follows:

a) $A(>, i)=A(>, i-1) ; F(>, i-1)$

$$
A(<, i)=A(<, i-1) ; F(<, i-1)
$$

b) $B(>, i)=B(>, i-1) ; G(<) ; F(<, i-1)$

$$
B(<, i)=B(<, i-1) ; G(>) ; F(>, i-1)
$$

c) $C(>, i)=C(>, i-1) ; H(<) ; F(<, i-1)$

$$
C(<, i)=C(<, i-1) ; H(>) ; F(>, i-1)
$$

d) $D(>, i)=A(>, i-1) ; E(>, i-1)$

$$
D(<, i)=A(<, i-1) ; E(<, i-1)
$$

e) $E(>, i)=B(>, i-1) ; J(<) ; D(>, i-1) ; E(>, i-1)$

$$
E(<, i)=B(<, i-1) ; J(>) ; D(<, i-1) ; E(<, i-1)
$$

f) $F(>, i)=C(>, i-1) ; K(<) ; D(>, i-1) ; E(>, i-1)$

$$
F(<, i)=C(<, i-1) ; K(>) ; D(<, i-1) ; E(<, i-1)
$$

that is, six parametrized functions recursively calling each other. (Since $D(>, 0)$ and $D(<$, (i) are "no operation"'s which do not change anything we leave them out, cf. below.)

Proof. For a) through f) we prove one equality each; the other one is symmetric. For all $i>0$, with $\left[I_{I-1}\right]_{I-1}=\varepsilon$ for $i=1$ by definition:

a) For $x \neq[$ :

$$
\begin{aligned}
>\left[_{I}\right]_{I}\left[_{i+1} x\right. & =>\left[{ }_{I-1}\right]_{I-1}\left[{ }_{i}\right]_{i}\left[_{i+1} x\right. \\
& \stackrel{A(>, i-1)}{\rightleftharpoons}\left[{ }_{i}\left[{ }_{I-1}\right]_{I-1}>\right]_{i}\left[_{i+1} x\right. \\
& \stackrel{F(>, i-1)}{\rightleftharpoons}\left[{ }_{i+1}\left[_{i}\right]_{i}[I-1]_{I-1}>x=\left[_{i+1}[I]_{I}>x ;\right.\right.
\end{aligned}
$$


b) For $x \neq \mathrm{]}$ :

$$
\begin{aligned}
& x\left[{ }_{j}<{ }_{I}\right]_{I}\left[_{i+1}=x\left[_{j}<\left[{ }_{I-1}\right]_{I-1}\left[_{i}\right]_{i}\left[_{i+1}\right.\right.\right. \\
& \stackrel{B(<, i-1)}{\Longrightarrow} x\left[\left[_{i}\left[{ }_{I-1}\right]_{I-1}>\left\{_{j}\right]_{i}\left[{ }_{i+1}\right.\right.\right. \\
& \stackrel{G(>)}{\Longrightarrow} x\left[{ }_{i}\left[{ }_{I-1}\right]_{I-1}>\right]_{i}\left[{ } _ { i + 1 } \left\{_{j}\right.\right. \\
& \stackrel{F(>, i-1)}{\rightleftharpoons} x\left[_{i+1}\left[{ }_{i}\right]_{i}\left[I_{I-1}\right]_{I-1}>\left\{_{j}\right.\right. \\
& =x\left[{ }_{i+1}\left[_{I}\right]_{I}>\left\{_{j}\right. \text {; }\right.
\end{aligned}
$$

c)

]$_{j+1}\left[_{j}<\left[_{I}\right]_{I}\left[_{i+1}=\right]_{j+1}\left[{ }_{j}<[I-1]\right]_{I-1}\left[{ }_{i}\right]_{i}\left[_{i+1}\right.\right.$

$$
\begin{aligned}
& \stackrel{C(<, i-1)}{\rightleftharpoons}\left[{ }_{i}\left[I_{I-1}\right]_{I-1}>\right\}_{j+1}\left\{_{j}\right]_{i}\left[_{i+1}\right. \\
& \stackrel{H(>)}{\Longleftrightarrow}\left[{ }_{i}\left[I_{I-1}\right]_{I-1}>\right]_{i}\left[[ _ { i + 1 } \} _ { j + 1 } \left\{_{j}\right.\right. \\
& \stackrel{F(>, i-1)}{\rightleftharpoons}\left[{ }_{i+1}\left[{ }_{i}\right]_{i}\left[I_{I-1}\right]_{I-1}>\right\}_{j+1}\left\{_{j}\right. \\
& \left.=\left[_{i+1}[]_{I}\right]_{I}>\right\}_{j+1}\left\{{ }_{j} ;\right.
\end{aligned}
$$

d) For $x \neq \mathrm{I}$ :

$$
\begin{aligned}
>\left[_{I}\right]_{I} x & =>\left[{ }_{I-1}\right]_{I-1}\left[{ }_{i}\right]_{i} x \\
& \stackrel{A(>, i-1)}{\Longrightarrow}\left[{ }_{i}\left[I_{I-1}\right]_{I-1}>\right]_{i} x \\
& \left.\stackrel{E(>, i-1)}{\Longrightarrow}[]_{i}\right]_{i}\left[I_{I-1}\right]_{I-1}>x \\
& =[]_{I}>x ;
\end{aligned}
$$

e) For $x \neq \mathrm{]}$ :

$\left.\left.x\left[_{i+1}<[]_{I}\right]_{I}\right]_{i+1}=x\left[_{i+1}<[I-1]\right]_{I-1}\left[{ }_{i}\right]_{i}\right]_{i+1}$

$$
\begin{aligned}
& \stackrel{B(<, i-1)}{\rightleftharpoons} x\left[{ }_{i}\left[{ }_{I-1}\right]_{I-1}>\left\{\left\{_{i+1}\right]_{i}\right]_{i+1}\right. \\
& \stackrel{J(>)}{\rightleftharpoons} x\left[{ }_{i}\left[{ }_{I-1}\right]_{l-1}<\right]_{i}\left[{ }_{i+1}\right]_{i+1} \\
& \stackrel{D(<, i-1)}{\rightleftharpoons} x\left[{ }_{i}<\left[{ }_{I-1}\right]_{I-1}\right]_{i}\left[\left[_{i+1}\right]_{i+1}\right. \\
& \stackrel{E(<, i-1)}{\rightleftharpoons} x<[I-1]]_{I-1}[i]_{i}\left[_{i+1}\right]_{i+1} \\
& =x<\left[_{I}\right]_{I}\left[_{i+1}\right]_{i+1} ;
\end{aligned}
$$

f) For $x \neq \mathrm{]}$ :

$\left.x]_{i+2}\left[{ }_{i+1}<[I]_{I}\right]_{i+1}=x\right]_{i+2}\left[{ }_{i+1}<\left[_{I-1}\right]_{I-1}\left[{ }_{i}\right]_{i}\right]_{i+1}$

$$
\stackrel{C(<, i-1)}{\rightleftharpoons} x\left[\left[_{i}[I-1]_{I-1}>\right\}_{i+2}\left\{{ }_{i+1}\right]_{i}\right]_{i+1}
$$




$$
\begin{aligned}
& \left.\stackrel{K(>)}{\Longleftrightarrow} x\left[{ }_{i}[I-1]_{I-1}<\right]_{i}[i+1]_{i+1}\right]_{i+2} \\
& \stackrel{D(<, i-1)}{\rightleftharpoons} x\left[{ }_{i}<\llbracket_{I-1} \rrbracket_{I-1} \rrbracket_{i}[i+1\rfloor_{i+1}\right\rfloor_{i+2} \\
& \left.\stackrel{E(<, i-1)}{\rightleftharpoons} x<[I-1]_{I-1}\left[{ }_{i}\right]_{i}[i+1]_{i+1}\right]_{i+2} \\
& \left.\left.=x<{ }_{I}\right\rfloor_{I}\left[{ }_{i+1}\right\rfloor_{i+1}\right\rfloor_{i+2} \text {. }
\end{aligned}
$$

The mappings $D(>, 0):>x \mapsto>x(x \neq[)$ and $D(<, 0): x<\mapsto x<(x \neq 1)$ are "no operation" or "skip" instructions. Deleting them henceforth in the expansion rules of Lemma $2 \mathrm{e}$ ) and $2 \mathrm{f}$ ), for $i=1$, those become:

$$
\begin{aligned}
& \text { ad Lemma 2a) } E(>, 1)=B(>, 0) ; J(<) ; E(>, 0) \\
& E(<, 1)=B(<, 0) ; J(>) ; E(<, 0) \\
& \text { ad Lemma 2f) } F(>, 1)=C(>, 0) ; K(<) ; E(>, 0) \\
& F(<, 1)=C(<, 0) ; K(>) ; E(<, 0) .
\end{aligned}
$$

A level $i$ expansion of a function $X(>, j)$ or $X(<, j), j \geqq i \geqq 0$, results from expanding that parametrized function with parameter $j$ into a sequence of parameter $i$ functions and nonparametrized functions, according to Lemma 2 (with the "no operation"'s $D(>, 0)$ and $D(<, 0)$ left out in case $i=0)$. So if $Y_{1}^{(i)} ; Y_{2}^{(i)} ; \cdots ; Y_{n}^{(i)}$ is a level $i$ expansion of $X(\cdot)$ then $X(\cdot)=Y_{1}^{(i)} ; Y_{2}^{(i)} ; \cdots ; Y_{n}^{(i)}$ with $Y_{l}^{(i)} \in\{A(>, i), A(<, i)$, $B(>, i), B(<, i), \cdots, F(<, i), G(>), G(<), \cdots, K(<)\}-\{D(>, 0), D(<, 0)\}, 1 \leqq l \leqq$ $n$. We extend the concept in the obvious way to sequences of functions $X_{1}\left(\diamond_{1}, j_{1}\right)$; $X_{2}\left(\diamond_{2}, j_{2}\right) ; \cdots ; X_{m}\left(\diamond_{m}, j_{m}\right), j_{1}, j_{2}, \cdots, j_{m} \geqq i$ and $\diamond_{1}, \diamond_{2}, \cdots, \diamond_{m} \in\{<,>\}$. For example, the level 0 expansion of $A(>, 3)$ is found by way of the level 2 and level 1 expansions:

$$
\begin{aligned}
A(>, 3)= & A(>, 2) ; F(>, 2) \\
= & A(>, 1) ; F(>, 1) ; C(>, 1) ; K(<) ; D(>, 1) ; E(>, 1) \\
= & A(>, 0) ; F(>, 0) ; C(>, 0) ; K(<) ; E(>, 0) ; C(>, 0) ; H(<) ; \\
& F(<, 0) ; K(<) ; A(>, 0) ; E(>, 0) ; B(>, 0) ; J(<) ; E(>, 0) .
\end{aligned}
$$

The atomic mappings of the level 0 expansions of the parametrized functions are called the local rewriting rules, and govern the switching of the tagged cells, in the squares scanned, by the basic steps of the oblivious one-head tape unit $M$. Note that a fat head covering four squares left and four squares right of the displayed center " $>$ " or " $<$ " suffices to execute these atomic mappings. Below we use superscripts to distinguish the identity of the various tagged cells before and after rewriting.

$$
\begin{aligned}
& \text { Local rewriting rules: } \\
& \begin{array}{l}
G(>):>\left\{{ }^{1}\right]^{2}\left[^{3} \mapsto>\right]^{2} I^{3}\left\{^{1}\right. \\
\left.\left.G(<):]^{1}\left[^{2}\right\}^{3}<\mapsto\right\}^{3}\right]^{1}\left[^{2}<\right. \\
H(>):>\}^{1}\left\{^{2}\right]^{3}\left[^{4} \mapsto>\right]^{3}\left[{ }^{4}\right\}^{1}\left\{^{2}\right. \\
H(<):]^{1}\left[{ }^{2}\right\}^{3}\left\{{ }^{4}<\mapsto\right\}^{3}\left\{^{4}\right]^{1} I^{2}< \\
\left.J(>):>\left\{\left\{^{1}\right]^{2}\right]^{3} \mapsto<\right]^{2}\left[^{1}\right]^{3} \\
J(<):\left[{ }^{1}\left[^{2}\right\}^{3}<\mapsto\left[^{1}\right]^{3}\left[^{2}>\right.\right.
\end{array}
\end{aligned}
$$




$$
\begin{aligned}
& \left.\left.\left.K(>):>\}^{1}\left\{{ }^{2}\right]^{3}\right]^{4} \mapsto<\right]^{3}\left[{ }^{2}\right]^{4}\right]^{1} \\
& K(<):\left[{ } ^ { 1 } [ ^ { 2 } \} ^ { 3 } \left\{{ }^{4}<\mapsto\left[{ } ^ { 4 } [ { } ^ { 1 } ] ^ { 3 } \left[{ }^{2}>\right.\right.\right.\right. \\
& A(>, 0):>[\mapsto[> \\
& A(<, 0):]<\mapsto<\text { ] } \\
& \left.\left.\left.B(>, 0):]^{1}>\right]^{2} x \mapsto\right\}^{2}<\right]^{1} x \quad(x \neq[) \\
& B(<, 0): x\left[^{1}<\left[^ { 2 } \mapsto x \left[^{2}>\left\{^{1} \quad(x \neq]\right)\right.\right.\right. \\
& \left.C(>, 0):]^{1}>\right]^{2}\left[\left[^{3} \mapsto\right\}^{2}\left\{^{3}<\right]^{1}\right. \\
& C(<, 0):]^{1}\left[{ }^{2}<{ }^{3} \mapsto\left[^{3}>\right\}^{1}\left\{^{2}\right.\right. \\
& E(>, 0):\left[{ }^{1}>\right]^{2} x \mapsto\left[{ }^{1}\right]^{2}>x \quad(x \neq[) \\
& E(<, 0): x\left[\left[^{1}<\right]^{2} \mapsto x<\left[^{1}\right]^{2} \quad(x \neq]\right) \\
& F(>, 0):\left[{ }^{1}>\right]^{2}\left[{ } ^ { 3 } \mapsto \left[{ }^{3}\left[{ }^{1}\right]^{2}>\right.\right. \\
& \left.F(<, 0):]^{1}\left[{ }^{2}<\right]^{3} \mapsto<\left[^{2}\right]^{3}\right]^{1}
\end{aligned}
$$

The only use of the context symbols $x$ in the definitions of $A(>, i), A(<, i), F(>, i)$ and $F(<, i)$ was to force a unique expansion into functions with parameter $j, j<i$, according to Lemma 2. Since $A(<, 0), A(>, 0), F(<, 0)$ and $F(>, 0)$ are atomic indivisible actions, because the local rewriting rules shall not be decomposed any further, we do not need these context symbols at the lowest level.

In the sequel it is useful to talk about well formed arrays, that is, the set of arrays from which the consecutive i.d.'s of $M$ are taken.

(i) $T^{0}$ is a well formed array.

(ii) If $T$ is a well formed array and $X($.$) is any local rewriting rule, with the dot$ standing for any appropriate argument, such that $T \stackrel{X(.)}{\Longleftrightarrow} T^{\prime}, T^{\prime} \neq \varnothing$, then $T^{\prime}$ is a well formed array.

(iii) No array is well formed except by (i) and (ii).

Since no mapping either deletes or multiplies a headmarker, i.e., "<" or " $>$ ", all well formed arrays contain a single headmarker. By the mutual exclusion of the subarrays they rewrite, if a well formed array $T$ is rewritten to $T^{\prime} \neq \varnothing$ by a local rewriting rule, then $T$ is rewritten to $\varnothing$ by all other local rewriting rules. We now show that a well formed array $T$ is always rewritten by some local rewriting rule to a another well formed array, which rewriting rule and array are therefore unique.

Earlier, we observed that, for all $i \geqq 0$,

$$
T^{0} \stackrel{A(>, i)}{\rightleftharpoons} T^{t_{i}}
$$

If $Y_{1}^{(0)} ; Y_{2}^{(0)} ; Y_{3}^{(0)} ; \cdots ; Y_{n}^{(0)}=A(>, i)$ is the level 0 expansion of $A(>, i)$ then, by Lemma 2, there exist well formed arrays $T_{0}^{(0)}, T_{1}^{(0)}, T_{2}^{(0)}, \cdots, T_{n}^{(0)}, T_{0}^{(0)}=T^{0}$ and $T_{n}^{(0)}=T^{t_{i}}$, such that

$$
T_{j-1}^{(0)} \stackrel{Y_{j}^{(0)}}{\Longrightarrow} T_{j}^{(0)}
$$

for all $j, 1 \leqq j \leqq n$. By the uniqueness of application of local rewriting rules it follows that

$$
T_{j-1}^{(0)} \stackrel{x}{\Rightarrow} \varnothing
$$


for all $j, 1 \leqq j \leqq n, X \neq Y_{j}^{(0)}$ and $X$ is a local rewriting rule. Hence each well formed array in the sequence $T_{0}^{(0)}, T_{1}^{(0)}, \cdots, T_{n-1}^{(0)}$ has exactly one local rewriting rule which is applicable to it, and the application of this local rewriting rule yields exactly one next well formed array. to

By Lemma 2a) we have $A(>, i)=A(>, i-1) ; F(>, i-1)$ for all $i>0$, which leads

$$
A(>, i)=A(>, 0) ; F(>, 0) ; F(>, 1) ; \cdots ; F(>, i-1)
$$

with

$$
T^{0} \stackrel{A(>, 0)}{\Longleftrightarrow} T^{t_{0}}
$$

and

$$
T^{t_{j}} \stackrel{F(>, j)}{\Longleftrightarrow} T^{t_{j+1}}
$$

for all $j, 0 \leqq j<i$. Define $A(>, \infty)$ by

$$
A(>, \infty)=\lim _{i \rightarrow \infty} A(i)=A(>, 0) ; F(>, 0) ; F(>, 1) ; \cdots ; F(>, i) ; \cdots
$$

and the level 0 expansion of $A(>, \infty)$ as the infinite, or unbounded, sequence of local rewriting rules resulting from the level 0 expansions of the constituent functions $F(>, i)$, $i>0$, above. So

$$
\begin{aligned}
A(>, \infty) & =Y_{1}^{(0)} ; Y_{2}^{(0)} ; \cdots ; Y_{i}^{(0)} ; \cdots \\
& =A(>, 0) ; F(>, 0) ; C(>, 0) ; K(<) ; E(>, 0) ; \cdots
\end{aligned}
$$

and there exists an infinite sequence of well formed arrays $T_{0}^{(0)}$, $T_{1}^{(0)}, \cdots, T_{i}^{(0)}, \cdots, T_{0}^{(0)}=T^{0}$, such that for all $j \geqq 1$

$$
T_{j-1}^{(0)} \stackrel{Y_{j}^{(0)}}{\Longleftrightarrow} T_{j}^{(0)}
$$

and for no local rewriting rule $X \neq Y_{j}^{(0)}$ and $T \neq \varnothing$

$$
T_{j-1}^{(0)} \stackrel{x}{\Rightarrow} T,
$$

i.e., $Y_{j}^{(0)}$ is the only local rewriting rule applicable to $T_{j-1}^{(0)}$. Consequently, a machine which wants to execute the sequence of local rewritings of the level 0 expansion of $A(>, \infty)$, starting with i.d. $T^{0}$, needs only to select the single local rewriting rule $Y_{j}^{(0)}$, applicable to the current $T_{j-1}^{(0)}$, by considering the length 9 subarray of $T_{j-1}^{(0)}$ with the current headmarker in the center, to obtain the next $T_{j}^{(0)}, j \geqq 1$. From the expansions in Lemma 2 we see that a nonparametrized function of $G, H, J, K$ is always followed by a parametrized function from $A, B, C, E, F$ in the level 0 expansion of $A(>, \infty)$. In a single step of $M$ we shall first execute a local rewriting according to $G, H, J$, or $K$, if possible, and then execute a local rewriting according to $A, B, C, E$ or $F$, which by the above is always possible, starting with initial i.d. $T^{0}$. So the oblivious one-head tape unit $M$ at each step shall examine the squares around the headmarker, and switches tagged cells and head position amongst the scanned squares according to the only local rewriting rules applicable. Fig. 5 shows an initial segment of the sequence of well formed arrays $T_{0}^{(0)}, T_{1}^{(0)}, \cdots, T_{i}^{(0)}, \cdots$ produced by the successive execution of the local rewriting rules in the level 0 expansion of $A(>, \infty)$ using the simple procedure SWITCH below. 


\begin{tabular}{|c|c|c|c|}
\hline Start i.d. & $>[][][][][][][][][][] \ldots$ & $=\mathrm{K}(<) \Longrightarrow$ & {$[[][[[][>]]]][][][][]$.} \\
\hline$\vDash A(>, 0) \Rightarrow$ & {$[>][][][][][][][][][] \ldots$} & $F \mathrm{E}(>, 0) \Rightarrow$ & {$[[][[[][]>]]][][][][]$. } \\
\hline$F F(>, 0) \Rightarrow$ & {$[[]>][][][][][][][][] \ldots$} & $\vDash \mathrm{B}(>, 0) \Rightarrow$ & $[[][[[][\}<]]][][][]][]$. \\
\hline$F C(>, 0) \Rightarrow$ & {$[[\}\langle<]][][][][][][][] \ldots$} & $=\mathrm{G}(<) \Longrightarrow$ & {$[[][[[\}][<]]][][][][]$.} \\
\hline$\vDash \mathrm{K}(<) \Longrightarrow$ & {$[[][>]][][][][][][][] \ldots$} & $=\mathrm{F}(<, 0) \Rightarrow$ & {$[[][[[]<[]]]][][][][]$.} \\
\hline$=E(>, 0) \Rightarrow$ & {$[[][]>][][][][][][][] \ldots$} & $F J(<) \Longrightarrow$ & {$[[][[][>[]]]][][][][]$.} \\
\hline$=C(>, 0) \Rightarrow$ & {$[[][\}\{<]][][][][][][] \ldots$} & $=\mathrm{A}(>, 0) \Rightarrow$ & {$[[][[][[>]]]][][][][]$.} \\
\hline $\mathrm{FH}(<) \Longrightarrow$ & {$[[\}\{][<]][][][][][][] \ldots$} & $=\mathrm{E}(>, 0) \Rightarrow$ & [[][i][[]>]]][][][][]. \\
\hline$F F(<, 0) \Rightarrow$ & {$[[\}\{<[]]][][][][][][] \ldots$} & $=\mathrm{B}(>, 0) \Rightarrow$ & {$[[][[][[\}<]]][][][][]$.} \\
\hline$=\mathrm{K}(<) \Longrightarrow$ & {$[[][>[]]][][][][][][] \ldots$} & $\vDash \mathrm{J}(<) \Longrightarrow$ & {$[[][[][][>]]][][][][]$.} \\
\hline$F \mathrm{~A}(>, 0) \Rightarrow$ & {$[[][[>]]][][][][][][] \ldots$} & $=E(>, 0) \Rightarrow$ & {$[[][[][][]>]][][][][]$.} \\
\hline$F \mathrm{E}(>, 0) \Rightarrow$ & {$[[][[]>]][][][][][][]$.} & $\vDash \mathrm{B}(>, 0) \Rightarrow$ & {$[[][[][][\}<]][][][][]$} \\
\hline$\vDash B(>, 0) \Rightarrow$ & $[[][1\}<]][][][][][][]$. & $\vDash G(<) \Longrightarrow$ & $[[][[][\}][<]][][][]][]$. \\
\hline$F \mathrm{~J}(<) \Longrightarrow$ & {$[[][][>]][][][][][][]$.} & $F \mathrm{~F}(<, 0) \Rightarrow$ & $[[][[][\}<[]]][][]][][]$. \\
\hline$F E(>, 0) \Rightarrow$ & {$[[][][]>][][][][][][]$.} & $\vDash G(<) \Longrightarrow$ & $[[][[]][<[]]][]][][][]$. \\
\hline$=C(>, 0) \Rightarrow$ & {$[[][][\}\{<]][][][][][]$.} & $=\mathrm{C}(<, 0) \Rightarrow$ & {$[[][[\}[>\}\{]]][][][][]$.} \\
\hline $\mathrm{FH}(<) \Longrightarrow$ & {$[[][\}\{][<]][][][][][]$.} & $\vDash \mathrm{K}(>) \Longrightarrow$ & $[[][[][<][]]][][][][]]$. \\
\hline$F F(<, 0) \Rightarrow$ & $[[][\}\{<[]]][][][][\}]]$. & $=\mathrm{E}(<, 0) \Rightarrow$ & {$[[][[]<[][]]][][][][]$.} \\
\hline$=\mathrm{H}(<) \Longrightarrow$ & $[[\}\{][<1]]][][][][][]$. & $\vDash \mathrm{J}(<) \Longrightarrow$ & {$[[][][>[][]]][][][][]$.} \\
\hline$F C(<, 0) \Rightarrow$ & {$[[\}\{[>\}\{]]][][][][][]$} & $=\mathrm{A}(>, 0) \Rightarrow$ & {$[[][][[>][]]][][][][]$.} \\
\hline$F \mathrm{~K}(>) \Longrightarrow$ & {$[[\}\{[<][]]][][][][][] \ldots$} & $F F(>, 0) \Rightarrow$ & {$[[][][[[]>]]][][][][]$.} \\
\hline$=E(<, 0) \Rightarrow$ & $[[\}\{<[]]]]][][][][][] \ldots$ & $=\mathrm{B}(>, 0) \Rightarrow$ & {$[[][][[[\}<]]][][][][]$.} \\
\hline$=\mathrm{K}(<) \Longrightarrow$ & {$[[][>[][]]][][][][][] \ldots$} & $\vDash \mathrm{J}(<) \Longrightarrow$ & {$[[][][[][>]]][][][][]$.} \\
\hline$F A(>, 0) \Rightarrow$ & {$[[][[>][]]][][][][][] \ldots$} & $=\mathrm{E}(>, 0) \Rightarrow$ & {$[[][][[][]>]][][][][]$.} \\
\hline$F F(>, 0) \Rightarrow$ & {$[[][[[]>]]][][][][][]$.} & $F \mathrm{~B}(>, 0) \Rightarrow$ & {$[[][][[][\}<]][][][][]$.} \\
\hline$=\mathrm{B}(>, 0) \Rightarrow$ & $[1][[[\}<]]][][][][][]$ & $F \mathrm{G}(<) \Longrightarrow$ & {$[[][][[\}][<]][][][][]$.} \\
\hline$F \mathrm{~J}(<) \Longrightarrow$ & {$[[][[][>]]][][][][][]$} & $F \mathrm{~F}(<, 0) \Rightarrow$ & {$[[][][[]<[]]][][][][]$.} \\
\hline$F E(>, 0) \Rightarrow$ & {$[[][[][]>]][][][][][]$} & $\vDash J(<) \Longrightarrow$ & {$[[][][][>[]]][][][][]$.} \\
\hline$F \mathrm{~B}(>, 0) \Rightarrow$ & {$[[][[][\}<]][][][][][]$} & $=\mathrm{A}(>, 0) \Rightarrow$ & {$[[][][][[>]]][][][][]$.} \\
\hline $\mathrm{F} \mathrm{G}(<) \Longrightarrow$ & {$[[][[\}][<]][][][][][]$} & $=\mathrm{E}(>, 0) \Rightarrow$ & {$[[][][][[]>]][][][][]$.} \\
\hline$F F(<, 0) \Rightarrow$ & $[[][[\}<[]]][][][]][][] \ldots$ & $F \mathrm{~B}(>, 0) \Rightarrow$ & {$[[][][][[\}<]][][][][]$.} \\
\hline$\vDash \mathrm{J}(<) \Longrightarrow$ & {$[[][][>[]]][][][][][]$} & $F \mathrm{~J}(<) \Longrightarrow$ & {$[[][][][][>]][][][][]$.} \\
\hline$\vDash A(>, 0) \Rightarrow$ & {$[[][][[>]]][][][][][]$} & $F E(>, 0) \Rightarrow$ & {$[[][][][][]>][][][][]$.} \\
\hline$=\mathrm{E}(>.0) \Rightarrow$ & {$[[][][[]>]][][][][][]$} & $F C(>, 0) \Rightarrow$ & {$[[][][][][\}\{<]][][][]$.} \\
\hline$F \mathrm{~B}(>, 0) \Rightarrow$ & {$[[][][[\}<]][][][][][]$} & $\mathrm{F} H(<) \Longrightarrow$ & {$[[][][][\}\{][<]][][][]$.} \\
\hline$F^{\prime} J(<) \Longrightarrow$ & {$[[][][][>]][][][][][]$} & $F F(<, 0) \Rightarrow$ & $[[][][][\}\{<[]]][][]]]$. \\
\hline$F E(>, 0) \Rightarrow$ & {$[[][][][]>][][][][][] \ldots$} & $\mathrm{F} H(<) \Longrightarrow$ & $[[][]\}\{][<]]][][][]$. \\
\hline$F C(>, 0) \Rightarrow$ & {$[[][][][\}\{<]][][][][] \ldots$} & $F C(<, 0) \Rightarrow$ & {$[[][][\}\{[>\}\{]]][][][]$} \\
\hline $\mathrm{FH}(<) \Longrightarrow$ & {$[[][][\}\{][<]][][][][] \ldots$} & $F \mathrm{~K}(>) \Longrightarrow$ & {$[[][][\}\{[<][]]][][][]$} \\
\hline$F F(<, 0) \Rightarrow$ & {$[[][][\}\{<[]]][][][][] \ldots$} & $F \mathrm{E}(<, 0) \Rightarrow$ & ][\}$\{<[][]]][][][$ \\
\hline $\mathrm{FH}(<) \Longrightarrow$ & {$[[][\}\{][<[]]][][][][] \ldots$} & $\mathrm{FH}(<) \Longrightarrow$ & $i<[1]$ \\
\hline$=C(<, 0) \Rightarrow$ & {$[[][\}\{[>\}\{]]\}[][][][] \ldots$} & $F C(<, 0) \Rightarrow$ & [] [] \\
\hline$F \mathrm{~K}(>) \Longrightarrow$ & []$][\}\{[<][]]][][][][]$ & $=\mathrm{H}(>) \Longrightarrow$ & ] [ ] \\
\hline$F \mathrm{E}(<, 0) \Rightarrow$ & []$][\}\{<[][$ & $F F(>, 0) \Rightarrow$ & []$>\}\{$ \\
\hline$F H(<) \Longrightarrow$ & {$[1\}\{][<1]$} & $F \mathrm{~K}(>) \Longrightarrow$ & $\{[[]<][]$ \\
\hline$=C(<.0) \Rightarrow$ & {$[[\}\{[>\}\{][]]][][][][]$} & $F \mathrm{~A}(<, 0) \Rightarrow$ & {$[[][\}\{[[<]][]]][][][]$.} \\
\hline$F H(>) \Longrightarrow$ & $[>1[\}\{]]][][][][]$ & $F \mathrm{E}(<, 0) \Rightarrow$ & {$[[][\}\{[<[]][]]][][][]$.} \\
\hline$F F(>.0) \Rightarrow$ & {$[[\}\{[[]>\}\{]]][][][][]$} & $F \mathrm{~B}(<, 0) \Rightarrow$ & {$[[][\}\{[>\{]][]]$} \\
\hline$=\mathrm{K}(>) \Longrightarrow$ & {$[[\}\{[[]<][]]][][][][]$} & $F \mathrm{~J}(>) \Longrightarrow$ & {$[[][\}\{[<][][]]][][][]$.} \\
\hline$F \mathrm{~A}(<, 0) \Rightarrow$ & {$[[\}\{[[<]][]]][][][][]$} & $F \mathrm{E}(<, 0) \Rightarrow$ & {$[[][\}\{<[][][]]][][][]$.} \\
\hline$\vDash E(<.0) \Rightarrow$ & $[[\}\{[<]]][]][][][][]$ & $\mathrm{FH}(<) \Longrightarrow$ & {$[[\}\{][<[][][]]][][][]$.} \\
\hline$\vDash \mathrm{B}(<, 0) \Rightarrow$ & {$[[\}\{[>\{]][]]][][][][]$} & $\vDash C(<, 0) \Rightarrow$ & {$[[\}\{[>\}\{][][]]][][][]$.} \\
\hline$F \mathrm{~J}(>) \Longrightarrow$ & $[1\}\{[<][][]]][][][][]$ & $F \mathrm{H}(>) \Longrightarrow$ & {$[[\}\{[>][\}\{][]]][][][]$.} \\
\hline$=\mathrm{E}(<, 0) \Rightarrow$ & {$[[\}\{<[][][]]][][][][] \ldots$} & $F F(>, 0) \Rightarrow$ & \\
\hline$F \mathrm{~K}(<) \Longrightarrow$ & {$[[][>[][][]]][][][][] \ldots$} & $F \mathrm{H}(>) \Longrightarrow$ & {$[[\}\{[[]>][\}\{]$} \\
\hline$F \mathrm{~A}(>, 0) \Rightarrow$ & {$[[][[>][][]]][][][][]$.} & $F C(>, 0) \Rightarrow$ & {$[[\}\{[]\}\{<]\}\{]$} \\
\hline$F F(>, 0) \Rightarrow$ & {$[[][[[]>][]]][][][][] \ldots$} & $F \mathrm{~K}(<) \Longrightarrow$ & {$[[][>]\}\{$} \\
\hline$\vDash C(>, 0) \Rightarrow$ & & $F E(>, 0) \Rightarrow$ & \\
\hline
\end{tabular}

FIG. 5. The first 116 well formed arrays $T_{j}^{(0)}, 0 \leqq j<116$.

\section{Procedure SWITCH:}

Step 1. Examine the length 9 subarray, centered on the headmarker, of the current i.d. and switch tagged cells and headmarker position according to the single, if any, local rewriting rule from the $G, H, J, K$ rules which is applicable. The result is a well formed array $T$. 
Step 2. Examine the length 9 subarray, centered on the headmarker, of array $T$ from step 1, and switch tagged cells and headmarker position according to the single local rewriting rule from the $A, B, C, E, F$ rules which is applicable. The resultant well formed array is the next i.d.

LEMMA 3. Starting from the initial i.d. $T^{0}$, a one-head tape unit $M$, executing SWITCH at each single step, executes exactly the local rewriting rule sequence of the level 0 expansion of $A(>, \infty)$. For each $t>0$, in the first $t$ steps $M$ executes this sequence up to and including the th occurrence of a local rewriting rule of the $A, B, C, E$ or $F$ type.

The goal of introducing the present bracket manipulator was to scan the subarray " $\left[{ }_{i}\right]_{i}\left[{ }_{i+1}\right]_{i+1}$ " at least once in each interval of $2^{\Theta(i)}$ steps, $i \geqq 0$. We can express precisely what the $t$ th i.d. $T^{t}$ is. $T^{0}$ is the initial array at time $t=0$, and $T^{t}$ results from an execution of SWITCH on $T^{t-1}$, for all $t>0$. According to Lemma $3, t$ equals the number of occurrences of $A, B, C, E, F$-type local rewriting rules executed. We need to recognize " $\left[{ }_{i}\right]_{i}\left[{ }_{i+1}\right]_{i+1}$ " as being the correct sequence of cells, which, since the cells are tagged with nonindexed brackets in the manipulator proper, cannot go by way of identifying the individual cells. For this purpose, the next lemma establishes a topology for the well formed arrays. Before proceeding, we review a few facts about well formed arrays which are pertinent to the proof of that lemma. By definition, and the discussion preceding Lemma 3, the set of well formed arrays equals the set $\left\{T_{j}^{(0)} \mid j \geqq 0\right\}$ defined by the level 0 expansion of $A(>, \infty)$.

$$
A(>, \infty)=Y_{1}^{(0)} ; Y_{2}^{(0)} ; \cdots ; Y_{i}^{(0)} ; \cdots
$$

and for all $i \geqq 1$

$$
T_{i-1}^{(0)} \stackrel{Y_{i}^{(0)}}{\Longleftrightarrow} T_{i}^{(0)} \quad \text { with } T_{0}^{(0)}=T^{0}
$$

By the definition of the initial array $T^{0}$, and those of the various procedures, each well formed array contains exactly one symbol from $\{<,>\}$ and, for each $i \geqq 1$, exactly one symbol from $\left.\left\{\mathbb{L}_{i},\left\{_{i}\right\} \text { and exactly one symbol from }\{]_{i},\right\}_{i}\right\}$. Recall that the indices are not really there but serve to identify the individual cells for the reader by distinguishing between the individual attached tags.

If a well formed array $T$ contains a pair of adjacent brackets " $\left.{ }_{j}\right]_{k}$ " then $j=k$; if it contains " $]_{j} \amalg_{k}$ " then $k=j+1$ in case the headmarker is to the left and $j=k+1$ in case the headmarker is to the right. More precisely: Then:

Lemma 4. Let $T$ be a well formed array, and let $\diamond \in\{<,>\}$ and $\alpha, \beta, \gamma \in\{[,],\{,\}\}^{*}$.

(i) $T=\alpha \diamond \beta\left[_{j}\right]_{k} \gamma \Rightarrow k=j$;

$\left.T=\alpha \llbracket_{k}\right]_{j} \beta \diamond \gamma \Rightarrow k=j$;

(ii) $T=\alpha \diamond \beta]_{j}\left[_{k} \gamma \Rightarrow k=j+1\right.$;

$$
T=\alpha]_{k}\left[{ }_{j} \beta \diamond \gamma \Rightarrow k=j+1\right. \text {. }
$$

Proof. We basically prove the lemma by induction on the sequence of well former arrays $T_{j}^{(0)}$, as defined by the level 0 expansion of $A(>, \infty), j \geqq 0$. To do so, we conside the initial segments $T_{j}^{(0)}[0: 2(i+1)], j \geqq 0$ and $i \geqq 0$, in isolation and show by the clair below that they internally satisfy the lemma. Viz., in executing the level 0 expansi of $A(>, i)$ to obtain $T^{t_{i}}$ from $T^{0}$ the elements of the sequence of subarrays $T_{0}^{(0)}[0: 2($ 1)], $T_{1}^{(0)}[0: 2(i+1)], \cdots, T_{a(i)}^{(0)}[0: 2(i+1)]$, with $T_{0}^{(0)}=T^{0}$ and $T_{a(i)}^{(0)}=T^{t_{i}}$, will be shor to internally satisfy the lemma. Since during the execution of $A(>, i)$ the final segmer. 
$T[2(i+1): \infty]$ is not changed at all, and $T^{0}$ satisfies the lemma, the elements of the sequence of subarrays $T_{0}^{(0)}[2(i+1): \infty], T_{1}^{(0)}[2(i+1): \infty], \cdots, T_{a(i)}^{(0)}[2(i+1): \infty]$ do internally satisfy the lemma. Because we have an overlap of one symbol between $T_{j}^{(0)}[0: 2(i+1)]$ and $T_{j}^{(0)}[2(i+1): \infty]$ for all $j, 0 \leqq j \leqq a(i)$ with $T_{0}^{(0)}=T^{0}$ and $T_{a(i)}^{(0)}=T^{t_{i}}$, we can conclude that each well formed array $T_{j}^{(0)}, 0 \leqq j \leqq a(i)$, satisfies the lemma. Taking the limit for $i \rightarrow \infty$, that is, considering $A(>, \infty)$, it follows that the lemma holds for all well formed arrays.

Claim. Let, for all $i \geqq 0, X \in\{A, B, C, D, E, F\}, \diamond \in\{<,>\}$ and $T, T^{\prime}$ be well formed arrays such that $X(\diamond, i): T[p: q] \mapsto T^{\prime}[p: q]$, for some $p, q \geqq 0$ and $T \stackrel{X(\diamond, i)}{\rightleftharpoons} T^{\prime}$,
and let $Y_{l+1}^{(0)} ; Y_{l+2}^{(0)} ; \cdots ; Y_{l+x(i)}^{(0)}$ be the level 0 expansion of $X(\diamond, i)$ with $T_{l+j-1}^{(0)} \stackrel{Y_{i+j}^{(0)}}{\Longleftrightarrow} T_{l+j}^{(0)}$ for all $j, 1 \leqq j \leqq x(i)$ with $T_{l}^{(0)}=T$ and $T_{l+x(i)}^{(0)}=T^{\prime}$. Then, for all $j$, $l \leqq j \leqq l+x(i), T_{j}^{(0)}[p: q]$ internally satisfies the lemma.

Proof of claim. Base case $i=0$. Since for $i=0$ the procedures are essentially but the local rewriting rules, we only have to verify that in the definitions of the various functions the subarrays left and right of the arrow internally satisfy the lemma. Note that $\left[_{I}\right]_{I} \stackrel{\text { def }}{=} \varepsilon$ for $i=0$.

Induction. Assume, by way of contradiction, that for some $X(\diamond, i)$, with $X \in$ $\{A, B, C, D, E, F\}$ and $\diamond \in\{<,>\}$ and $i>0$, the claim does not hold. But in the execution of the level $i-1$ expansions of six of these functions with parameter $i$ in the proof of Lemma 2 , the other six cases being symmetrical, the displayed subarrays all satisfy the claim. Hence it must follow that a nondepicted subarray arising in the execution of the level 0 expansion of some $X^{\prime}\left(\diamond^{\prime}, i-1\right), X^{\prime} \in\{A, B, C, D, E, F\}$ and $\diamond^{\prime} \in\{<,>\}$, violates the claim. Regressing in this fashion all the way down to $i=0$, we contradict the established base case, and the claim is proven.

By the discussion preceding the claim we have established the lemma.

Lemma 5. Let $T$ be a well formed array and let $\diamond \in\{<,>\}$ and $\alpha, \beta \in\{[,],\{,\}\}^{*}$. Then

$$
\left(T=\alpha \diamond\left[_{j}\right]_{k} \beta \text { or } T=\alpha\left[\left[_{k}\right]_{j} \diamond \beta\right) \Rightarrow(k=j=1) .\right.
$$

Proof. That $k=j$ follows already from Lemma 4 . Considering the level 1 expansion of $A(>, \infty)$

$$
A(>, \infty)=Y_{1}^{(1)} ; Y_{2}^{(1)} ; \cdots ; Y_{j}^{(1)} ; \cdots
$$

and

$$
T_{j-1}^{(1)} \stackrel{Y_{j}^{(1)}}{\Longleftrightarrow} T_{j}^{(1)} \text { with } T_{0}^{(1)}=T^{0},
$$

we observe that it follows from the definitions of the various procedures that, for all well formed arrays $T_{j}^{(1)}, j \geqq 0$, the lemma holds. Expanding each $A, B, C, D, E, F$ function with parameter 1 to level 0 , and examining the intermediate well formed arrays $T_{j^{\prime}}^{(0)} \neq T_{j}^{(1)}, j, j^{\prime} \geqq 0$, yields the lemma.

Lemma 4 and Lemma 5 show that a certain topological connectedness between the indexed brackets is preserved throughout the array at all times, and that, in particular, in each well formed array $\left.\alpha \diamond \beta\left[{ }_{j}\right]_{k} L_{l}\right]_{m} \gamma$ holds $k=j$ and $l=m=j+1$. So whenever there occurs a length four subarray "[] []" right of the headmarker the tagged cells concerned are in the correct consecutive left to right order. Without further proof we give a more exhaustive characterization of the topology. Let $T$ be a well formed array. Then, for each $i \geqq 1, T$ satisfies precisely one of the following forms. 
For $i=1$ :

$$
\begin{aligned}
& \left.\alpha[]_{1}\right]_{1} \diamond \beta \\
& \alpha \diamond\left[_{1}\right]_{1} \beta \\
& \alpha\left[{ }_{1} \diamond\right]_{1} \beta \\
& \alpha\left\lfloor_{1}>\left\{_{j}\right\rfloor_{1}\left[_{2}\right]_{2} \cdots\left\lfloor_{j-1}\right]_{j-1}\right]_{j} \beta \quad \text { (some } j \geqq 2 \text { ) } \\
& \left.\alpha\left[_{1}>\right\}_{j+1}\left\{{ }_{j}\right]_{1}\left[_{2}\right]_{2} \cdots\left[_{j-1}\right]_{j-1}\right]_{j} \beta \quad \text { (some } j \geqq 2 \text { ) }
\end{aligned}
$$

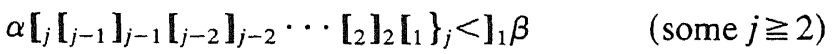

$$
\begin{aligned}
& \alpha\left[\left[_{j}\left[_{j-1}\right]_{j-1} L_{j-2}\right]_{j-2} \cdots I_{2}\right]_{2}\left[_{1}\right\}_{j}\left\{_{j+1}<\right]_{1} \beta \quad \text { (some } j \geqq 2 \text { ), }
\end{aligned}
$$

with the obvious modification for $j=2$.

For each $i>1$ :

$$
\begin{aligned}
& \alpha\left[_{i}\right]_{i}\left[{ }_{i-1} \beta \diamond \gamma\right. \\
& \alpha \diamond \beta]_{i-1}\left[{ }_{i}\right]_{i} \gamma \\
& \left.\alpha[i \beta \diamond \gamma\}_{i}\left\{_{i-1}\right]_{j}\left[_{j+1}\right]_{j+1}\left[{ }_{j+2}\right]_{j+2} \cdots\left[{ }_{i-2}\right]_{i-2}\right]_{i-1} \delta \quad \text { (some } j<i-1 \text { ) } \\
& \alpha \diamond \beta\left\{\left\{_{i}\right]_{j}\left[_{j+1}\right]_{j+1}\left[_{j+2}\right]_{j+2} \cdots\left[\left[_{i-1}\right]_{i-1}\right]_{i} \gamma \quad \text { (some } j \leqq i-1\right. \text { ) } \\
& \alpha\left[{ }_{i-1}\left[{ }_{i-2}\right]_{i-2}\left[_{i-3}\right]_{i-3} \cdots\left[_{j+1}\right]_{j+1} L_{j}\right\}_{i-1}\left\{\left\{_{i} \beta \diamond \gamma\right]_{i} \delta \quad \text { (some } j<i-1\right. \text { ) } \\
& \alpha\left[\left[_{i}\left[{ }_{i-1}\right]_{i-1}\left[_{i-2}\right]_{i-2} \cdots\left[_{j+1}\right]_{j+1} L_{j}\right\}_{i} \beta \diamond \gamma \quad \text { (some } j \leqq i-1\right. \text { ), }
\end{aligned}
$$

with the obvious modification for $i-3 \leqq j \leqq i-1$. Here $\diamond$ can be either " $<$ " or " $>$ " and $\alpha, \beta, \gamma, \delta \in\{[,],\{,\}\}^{*}$. Considering the fact that

$$
T^{0}=>\left[{ }_{1}\right]_{1}\left[{ }_{2}\right]_{2} \cdots\left[{ }_{i}\right]_{i}\left[{ }_{i+1}\right]_{i+1} \cdots
$$

and that, by definition, for $i \geqq 0$ and $t \leqq t_{i}$,

$$
\left.T^{t}=\alpha \diamond \beta\right]_{i+1}\left[_{i+2}\right]_{i+2} \cdots\left[{ }_{i+j}\right]_{i+j}\left[_{i+j+1}\right]_{i+j+1} \cdots,
$$

$j \geqq 3$, the formats express, but for the choice of $\diamond$ as " $<$ " or " $>$ ", the format each well formed array $T^{t}$ can have, by applying in sequence the requirements for $i+$ $1, i, \cdots, 1$. According to Lemma 4, whenever we scan a subarray "[] []" right of the headmarker, we know for sure that this is the subarray " $\left[{ }_{i}\right]_{i}\left[{ }_{i+1}\right]_{i+1}$ " for some $i \geqq 1$. In the next lemma we give an upper bound on the number of steps, that is, executions of SWITCH, in between scanning " $\left[{ }_{i}\right]_{i}\left[{ }_{i+1}\right]_{i+1}$ " right of the headmarker, for all $i \geqq 1$. To express the timing we consider expansions of $A(>, \infty)$ of level $i, i \geqq 1$ :

$$
A(>, \infty)=Y_{1}^{(i)} ; Y_{2}^{(i)} ; \cdots ; Y_{j}^{(i)} ; \cdots
$$

and define for all $j \geqq 1$

$$
T_{j-1}^{(i)} \stackrel{Y_{j}^{(i)}}{\Longleftrightarrow} T_{j}^{(i)} \text { with } T_{0}^{(i)}=T^{0} .
$$

The level 0 expansion of $Y_{j}^{(i)}=X(\diamond, i)$, with $X \in\{A, B, C, D, E, F\}$ and $\diamond \in$ $\{<,>\}$, is fixed and, but for the headmarker arguments, is the same whether $\diamond=<$ or $\diamond=>$. Thus, by Lemma 3 , the number of steps of $M$ to execute $X(\diamond, i)$ equals the number of occurrences of $A, B, C, E, F$ local rewriting rules in its level 0 expansion, and does not depend on the orientation of the headmarker $\diamond$, or the position $j$ in the level $i$ expansion of $A(>, \infty)$ where $Y_{j}^{(i)}$ occurs. We denote the number of steps, used by $M$, to execute $X(\diamond, i)$, by $T_{X}(i)$. 
LEMMA 6. There exists a function $S: \mathbb{N} \rightarrow \mathbb{N}$ such that for each $t \geqq 0$ there is a $t^{\prime}>t$ such that for some $\alpha, \alpha^{\prime}, \beta, \beta^{\prime} \in\{[,],\{,\}\}^{*}$ and $\diamond, \diamond^{\prime} \in\{<,>\}$

(i) $\left.\left.T^{t}=\alpha \diamond[]_{1}\right]_{1} \beta \Rightarrow T^{t^{\prime}}=\alpha^{\prime} \diamond^{\prime}[]_{1}\right]_{1} \beta^{\prime}$ and $t^{\prime}-t \leqq S(1)$.

(ii) $\left.\left.\left.T^{t}=\alpha \diamond[]_{1}\right]_{1} \coprod_{2}\right]_{2} \beta \Rightarrow T^{t^{\prime}}=\alpha^{\prime} \diamond^{\prime}\left[_{1}\right]_{1} \coprod_{2}\right]_{2} \beta^{\prime}$ and $t^{\prime}-t \leqq S(2)$.

(iii) For all $i>2$ and $x \in\left\{\varepsilon,\{\}\{\}\right.$, there is a $x^{\prime} \in\left\{\varepsilon,\{\}\{\}\right.$, such that: $T^{t}=$ $\left.\left.\alpha \diamond x]_{i-2}\left[{ }_{i-1}\right]_{i-1}[]_{i}\right]_{i} \beta \Rightarrow T^{t^{\prime}}=\alpha^{\prime} \diamond^{\prime} x^{\prime}\right]_{i-2}\left[{ }_{i-1}\right]_{i-1}\left[{ }_{i}\right]_{i} \beta^{\prime}$ and $t^{\prime}-t \leqq S(i)$.

Moreover, $S(i)=2 T_{A}(i)+T_{F}(i)$, for all $i \geqq 1$, is such a function.

Proof. Consider the level $i$ expansion of $A(>, \infty), i \geqq 1$,

$$
A(>, \infty)=Y_{1}^{(i)} ; Y_{2}^{(i)} ; \cdots ; Y_{j}^{(i)} ; \cdots
$$

and

$$
T_{j-1}^{(i)} \stackrel{Y_{i}^{(i)}}{\Longleftrightarrow} T_{j}^{(i)} \quad \text { with } T_{0}^{(i)}=T^{0}
$$

Then $T_{j}^{(i)}$ is of the form $\alpha \diamond\left[_{I}\right]_{I} \beta$ or $\alpha\left[_{I}\right]_{I} \diamond \beta$, for all $j \geqq 0$. All such $T_{j}^{(i)}$ 's, with $Y_{j}^{(i)}$ not $G, H, J$ or $K$ local rewriting rules, are i.d.'s of $M$. We restrict attention to the particular subsequence $T_{j_{0}}^{(i)}, T_{j_{1}}^{(i)}, \cdots, T_{j_{k}}^{(i)}, \cdots$ for which $T_{j_{k}}^{(i)}$ is of the form $\alpha<\mathrm{I}_{I} \mathrm{~J}_{I} \beta$ for all $k>0$ and $T_{j_{0}}^{(i)}=T^{0}$. For each $k \geqq 0$ there exists a sequence $Y_{j_{k}+1}^{(i)}$; $Y_{j_{k}+2}^{(i)} ; \cdots ; Y_{j_{k+1}}^{(i)}$ such that

$$
T_{j_{k}}^{(i)} \stackrel{Y_{j_{k}+1}^{i} ; Y_{j_{k}+2}^{i} ; \cdots ; Y_{j_{k+1}}^{i}}{\Longrightarrow} T_{j_{k+1}}^{(i)} .
$$

By the use of the recursive expressions in Lemma 2 we can determine all such sequences. Subsequently, we have to determine which such sequences take the most steps to execute. So we first determine $T_{X}(i)$ for all $X \in\{A, B, C, D, E, F\}$. It follows from Lemma 3 that $T_{X}(i)$ equals the number of occurrences of $A, B, C, E, F$ procedures in its level 0 expansion. We see from Lemma 2 that:

$$
\begin{aligned}
& T_{A}(i)=T_{A}(i-1)+T_{F}(i-1), \\
& T_{B}(i)=T_{B}(i-1)+T_{F}(i-1), \\
& T_{C}(i)=T_{C}(i-1)+T_{F}(i-1), \\
& T_{D}(i)=T_{A}(i-1)+T_{E}(i-1), \\
& T_{E}(i)=T_{B}(i-1)+T_{D}(i-1)+T_{E}(i-1), \\
& T_{F}(i)=T_{C}(i-1)+T_{D}(i-1)+T_{E}(i-1),
\end{aligned}
$$

nd $T_{A}(0)=T_{B}(0)=T_{C}(0)=T_{E}(0)=T_{F}(0)=1$ and $T_{D}(0)=0$. For this system of zurrence equations with initial values we find $T_{A}(i)=T_{B}(i)=T_{C}(i)$, for all $i \geqq 0$, $\mathrm{d}$ consequently $T_{E}(i)=T_{F}(i)$, for all $i \geqq 0$, which in its turn yields $T_{D}(i)=T_{A}(i)$, all $i \geqq 1$. Hence for all $i \geqq 1$ :

$$
T_{E}(i)=T_{F}(i) \geqq T_{A}(i)=T_{B}(i)=T_{C}(i)=T_{D}(i) .
$$

ow let $Y_{j_{k}+1}^{(i)} ; Y_{j_{k}+2}^{(i)} ; \cdots ; Y_{j_{k+1}}^{(i)}$ be a sequence of functions as in (1). Erasing the $j, H, J$ and $K$ procedures (because they do not contribute to the number of steps it takes to execute this sequence, by Lemma 3 ) and replacing all $E$ 's by $F$ 's and all $B$ 's, $C$ 's and $D$ 's by $A$ 's (because they take the same number of steps for $i \geqq 1$ ) the resulting sequences are $F(<, i), A(<, i) ; A(>, i)$ and $A(<, i) ; F(>, i) ; A(<, i)$. So for $i=1,2$, $S(i)=2 T_{A}(i)+T_{F}(i)$ satisfies the lemma. For $i>2$ we note that, for all $k \geqq 0$ (with 
the obvious modification for $k=0$ ),

$$
\begin{aligned}
& T_{j_{k}}^{(i)}=\alpha<[I-3\rfloor_{I-3}\left\lfloor_{i-2}\right\rfloor_{i-2}\left\lfloor_{i-1}\right\rfloor_{i-1}\left\lfloor_{i}\right]_{i} \beta \\
& \stackrel{z_{i}}{\Longleftrightarrow} \alpha^{\prime}\left[{ }_{i-2}[I-3]_{I-3}>x\right]_{i-2}\left[{ }_{i-1}\right]_{i-1}[i]_{i} \beta
\end{aligned}
$$

with $Z_{i}$ and $x$ one of the following:

$$
\begin{aligned}
& Z_{i} \in\{J(<) ; A(>, i-3), K(<) ; A(>, i-3)\} \quad \text { and } x=\varepsilon ; \\
& Z_{i}=B(<, i-3) \text { and } x=\{ \\
& \left.Z_{i} \in\{C(<, i-3), G(<) ; C(<, i-3), H(<) ; C(<, i-3)\} \text { and } x=\right\}\{.
\end{aligned}
$$

In all cases, the execution time of $Z_{i}$ is $T_{A}(i-3)$, which shows that $S(i)=2 T_{A}(i)+T_{F}(i)$ satisfies the lemma for all $i>2$ too.

COROllary. Let $S: \mathbb{N} \rightarrow \mathbb{N}$ be defined by $S(i)=2 T_{A}(i)+T_{F}(i)$, for all $i \geqq 1$. Then:

(i) For each $t \geqq 0$ there exists a $t^{\prime}, t<t^{\prime} \leqq t+S(1)$, such that the $t^{\prime} t h$ i.d. of $M$ has the form $T^{t^{\prime}}=\alpha<\left[_{1}\right]_{1} \beta$ for some $\alpha, \beta \in\{[,],\{,\}\}^{*}$.

(ii) For each $t \geqq 0$ there exists a $t^{\prime}, t<t^{\prime} \leqq t+S(2)$, such that the $t^{\prime} t h$ i.d. of $M$ has the form $T^{t^{\prime}}=\alpha<\left[_{1}\right]_{1}\left[_{2}\right]_{2} \beta$ for some $\alpha, \beta \in\{[,],\{,\}\}^{*}$.

(iii) For each $i>2$ and $t \geqq 0$ there exists a $t^{\prime}, t<t^{\prime} \leqq t+S(i)$, such that the $t^{\prime}$ th i.d. of $M$ has the form $\left.T^{t^{\prime}}=\alpha>x\right]_{i-2}\left[_{i-1}\right]_{i-1}\left[_{i}\right]_{i} \beta$ for some $\alpha, \beta \in\{[,]\{,\}\}^{*}$ and $x \in\{\varepsilon,\{\}\{\}$, .

It remains to determine $S$ analytically.

LEMMA 7. $S(i)=(1+\sqrt{2})^{i+1}+(1-\sqrt{2})^{i+1}$.

Proof. From the system of recurrence equations, and the values for $i=0$, in the proof of Lemma 6, follows:

$$
T_{A}(i)=2 T_{A}(i-1)+T_{A}(i-2) \text { for } i>2 .
$$

The solution for this homogeneous equation is of the form $T_{A}(i)=a x_{1}^{i}+b x_{2}^{i}$, where $x_{1,2}$ are the roots of $x^{2}-2 x-1=0$ and $a$ and $b$ follow from $T_{A}(1)=2$ and $T_{A}(2)=4$. So $x_{1,2}=1 \pm \sqrt{2}$ and

$$
a(1+\sqrt{2})+b(1-\sqrt{2})=2, \quad a(1+\sqrt{2})^{2}+b(1-\sqrt{2})^{2}=4
$$

yielding $a=1 / \sqrt{2}$ and $b=-1 / \sqrt{2}$. Hence

$$
T_{A}(i)=\frac{1}{\sqrt{2}}(1+\sqrt{2})^{i}-\frac{1}{\sqrt{2}}(1-\sqrt{2})^{i}, \quad i \geqq 1 .
$$

From the system of recurrence equations, and the identities amongst the functions, it appears that $T_{F}(i)=T_{A}(i)+T_{A}(i-1)$ whence the expression for $S(i)$ follows. $1+\sqrt{2}$.

COROLlaRY. $S(i)<3^{i+1}$ for all $i \geqq 1$. Viz., $S(1)=6$ and $\lim _{i \rightarrow \infty} S(i+1) / S(i)=$

Of course we can obtain that $S(i)<3^{i+1}$ by a cruder argument. The present analysis, however, is quite straightforward and precise. Running the bracket manipulator on a computer, by way of empirical verification, confirmed the first nine values of $S$.

2.4. The real-time simulator. Having set the stage in the preceding sections, we now tie everything together to obtain the desired real-time simulator.

Let $M$ be a one-head tape unit with a one-way infinite tape divided into two tracks: the tag track and the count track. The finite control of $M$ has a special register containing the initial segment $C[0: 5]$ of the array $C[0: \infty]$ representing the current 
count as in $\S \S 2.1-2.2$. The single head of $M$ covers 14 squares and its position is the intersquare boundary in the center. Initially, the head covers the leftmost squares, all squares on the tape contain special blank symbols and the finite control is in a distinguished initial state, in particular $C[0: 5]$ contains $\overline{0}$ 's only. Since $M$ can always initialize previously unscanned squares, still containing blanks, by keeping a parity bit in the finite control, we assume that the tape is initially divided in the two tracks as follows. Number the tape squares from left to right by $-7,-6, \cdots, 0,1,2, \cdots$. Square $i, i \geqq 0$, contains initially on the count track $C^{0}[i+6]=\overline{0}$ and on the tag track a tag "[", if $i$ is even, and a tag "]", if $i$ is odd. So the initial situation can be visualized as in Fig. 6, with the initial headmarker " $>$ " kept in the finite control. At each step the head rewrites the contents in the squares under scan, and shifts left, right or not at all. Since the head shifts will be governed by the local rewriting rules of the last section, the marker " $>$ " or " $<$ ", positioned on the center intersquare border of the scanned squares, can shift at most two squares left or right in a single step. Whether this marker is " $>$ " or " $<$ " can be maintained in the finite control; the initial marker is " $>$ ".

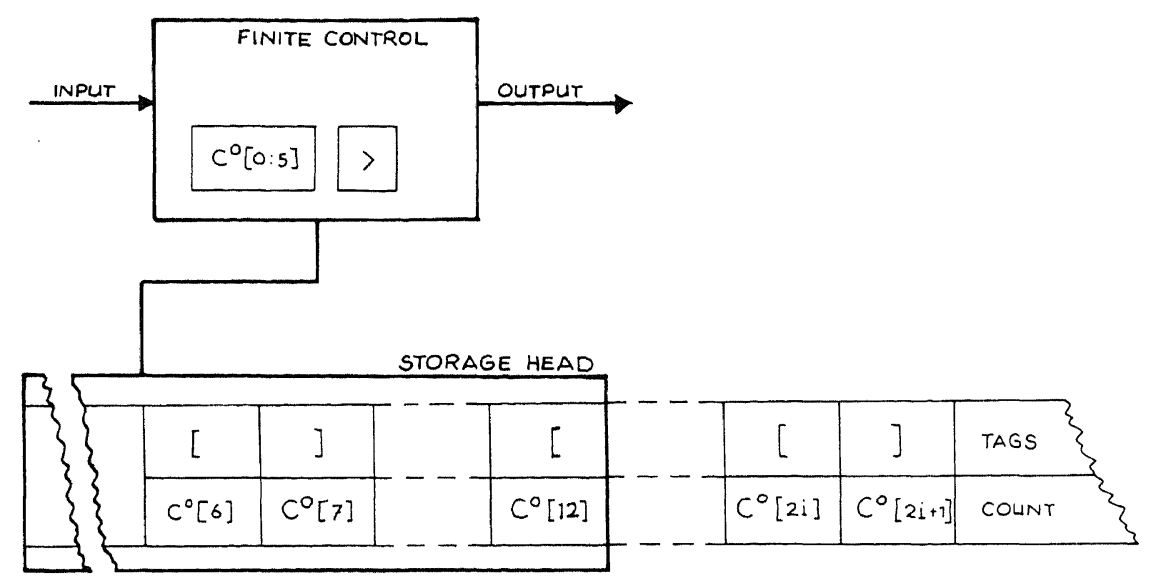

FIG. 6

Each step of $M$ consists of essentially two parts: first execute COUNT on the representation of the currently stored integer, check whether this integer is zero, and secondly execute SWITCH to switch cells containing digits of the integer representation. The information in the two tracks of a square may be thought of as a cell containing the current digit $C[i]$, which is tagged by the tag on the tag track.

To execute COUNT, $M$ inspects the scanned cells right of the headmarker, so as to determine $I(t)$ in the $t$ th step, and also identify the squares containing $C[2 i]$, $C[2 i+1], C[2 i+2]$ and $C[2 i+3]$ for all $i \in I(t)$. To this purpose first procedure COLLECT is executed. Let $P$ be the current local tape contents, i.e.

$$
P=\begin{array}{lllllll}
\tau_{1} & \tau_{2} & \tau_{3} & \tau_{4} & \tau_{5} & \tau_{6} & \tau_{7} \\
\gamma_{1} & \gamma_{2} & \gamma_{3} & \gamma_{4} & \gamma_{5} & \gamma_{6} & \gamma_{7}
\end{array}
$$

is the tape contents on the seven squares right of the headmarker.

Procedure COLLECT $(P)$ :

Let the seven squares right of the headmarker contain the string $\tau_{1} \tau_{2} \cdots \tau_{7}$ on the tag track and the string $\gamma_{1} \gamma_{2} \cdots \gamma_{7}$ on the count track. Then we distinguish 
essentially four cases, implicitly specified by $t$ :

(a) $\tau_{1} \tau_{2}=[] \& \tau_{3} \tau_{4} \neq[] \Rightarrow I(t)=\{0,1,2\} \& C[6: 7]=\gamma_{1} \gamma_{2}$

(b) $\tau_{1} \tau_{2} \tau_{3} \tau_{4}=[][] \Rightarrow I(t)=\{0,1,2,3\} \& C[6: 9]=\gamma_{1} \gamma_{2} \gamma_{3} \gamma_{4}$

(c) $\left.\tau_{1} \tau_{2} \tau_{3} \tau_{4} \tau_{5}=\right][][] \Rightarrow I(t)=\{0,1, i\}, i>3, \& C[2 i: 2 i+3]=\gamma_{2} \gamma_{3} \gamma_{4} \gamma_{5}$; $\tau_{1} \tau_{2} \tau_{3} \tau_{4} \tau_{5} \tau_{6}=\{][][] \Rightarrow I(t)=\{0,1, i\}, i>3, \& C[2 i: 2 i+3]$

$$
\begin{array}{r}
\left.\tau_{1} \tau_{2} \tau_{3} \tau_{4} \tau_{5} \tau_{6} \tau_{7}=\right\}\{][][] \Rightarrow I(t)=\{0,1, i\}, i>3, \& C[2 i: 2 i+3] \\
=\gamma_{3} \gamma_{4} \gamma_{5} \gamma_{6} \\
=\gamma_{4} \gamma_{5} \gamma_{6} \gamma_{7}
\end{array}
$$

(d) none of (a)-(c) $\Rightarrow I(t)=\{0,1\}$.

Modulo the correctness of the implications in the definition of COLLECT, which remain to be proven, the execution of COLLECT $(P)$ in the $t$ th step of $M$ both determines $I(t)=\left\{i_{l}, i_{l-1}, \cdots, i_{1}\right\}, i_{l}>i_{l-1}>\cdots>i_{1}$, and identifies the locations where $C\left[2 i_{j}\right], C\left[2 i_{j}+1\right], C\left[2 i_{j}+2\right]$ and $C\left[2 i_{j}+3\right]$ currently reside, $1 \leqq j \leqq l$. Since these locations are either under scan on the tape, or in the finite control, viz. $C[0: 5]$, the machine can in the $t$ th step execute COUNT $(t, \delta)=\operatorname{UPDATE}\left(i_{l}\right) ; \operatorname{UPDATE}\left(i_{l-1}\right) ; \cdots$; UPDATE $\left(i_{1}\right)$; INPUT $(\delta)$ by executing the consecutive mappings in the decomposition on the relevant subarrays of $C[0: \infty]$, without explicitly knowing the value of $t$. Thus, in each single step, starting from the all-blank tape with the initial headmarker " $>$ " positioned at the left end, the one-head tape unit $M$ will do all of the following.

Procedure STEP:

Step 1. Initialize both tracks of right adjacent previously unscanned squares, still containing primeval blanks, by writing the correct square bracket on the tag track (check and update parity count in the finite control) and a blank "D" in the count track of such a square.

Step 2. Execute COLLECT $(P)$.

Step 3. Let the current value of $I$ determined by step 2 be $\left\{i_{l}, i_{l-1}, \cdots, i_{1}\right\}$ with $i_{l}>i_{l-1}>\cdots>i_{1}$. READ the current value of $\delta$ from the input terminal and execute COUNT (current step, $\delta$ ), that is,

UPDATE $\left(i_{l}\right)$; UPDATE $\left(i_{l-1}\right) ; \cdots ; \operatorname{UPDATE}\left(i_{1}\right) ; \operatorname{INPUT}(\delta)$.

Step 4. WRITE "count equal zero" or "count unequal zero" to the output terminal, depending on whether or not $C[0]=\overline{0}$, for the $C[0]$ resulting from step 3.

Step 5. Execute SWITCH. That is, switch the contents of the scanned squares, considering the combined contents of the tag track and the count track on a square as a single package. Interchange these packages amongst squares, shift the head position and change the brackets and headmarker, governed by the current headmarker, head position on the scanned squares, and the scanned brackets alone.

PROPOSITION 4. The constructed one-head tape unit $M$ is oblivious and real-time simulates the quintessential counter.

Proof. The one-head tape unit $M$ is oblivious since the head movement is governed by the tag track and the headmarker, independent of the input. Attaching imaginary indexes $i=3,4, \cdots$ to the initial tag track contents, a shift of 2 from the ones in the initial i.d. in the previous section, the executions of SWITCH preserve that pairing of $C[2 i]$ with opening bracket indexed $i$ and of $C[2 i+1]$ with closing bracket indexed $i, i \geqq 3$. Since $C[0: 5]$ resides immobile in the finite control, Lemmas $2-5$ ensure that the identification of array elements by COLLECT $(P)$ in each step remains correct 
under the interchange of the mobile array elements of $C$ on the count track by SWITCH. In the $t$ th step, for all $t \geqq 1$, COLLECT $(P)$ determines the value $I(t)$ of the parameter selection function $I$, as well as the high to low order of elements in $I(t)$. By Lemma 6 and Corollary, for each $t \geqq 0$ and each index $i \geqq 2$, there exists a step $t^{\prime}$, $t<t^{\prime} \leqq t+S(i-1)$, such that $i \in I\left(t^{\prime}\right)$. By the definition of COLLECT $(P),\{0,1\} \subseteq I(t)$ for all $t \geqq 1$. Since it follows from Lemma 7 that $S(i-1)<3^{i}$ for all $i \geqq 2$, the oblivious one-head tape unit $M$ real-time simulates the quintessential counter by Propositions 2 and 3.

Let $C$ be any $k$-counter machine, $k \geqq 1$. Clearly, $C$ can be thought of as a finite control connected with $k$ quintessential counters $S_{1}, S_{2}, \cdots, S_{k}$. At each step the finite control of $C$ reads an input command from the input terminal if it is in a polling state, checks each $S_{i}, 1 \leqq i \leqq k$, for zero contents, and governed by this information issues input commands "add $\delta_{i}$ ", $\delta_{i} \in\{-1,0,1\}$, to each $S_{i}, 1 \leqq i \leqq k$, and writes an output string to the output terminal. In the spirit of Proposition 1, we can real-time simulate $C$ by an oblivious one-head tape unit $M_{C}$, which is just like $M$, but with $k$ count tracks (one for each quintessential counter) and one tag track. Storing the first six digits of the representation of each count in the finite control, which is connected to the input and output terminals through $C$ 's original finite control, we finally obtain.

THEOREM. Each multicounter machine can be real-time simulated by an oblivious one-head tape unit using logarithmic space.

Proof. By Propositions 1 and 4. That the space used is logarithmic in the simulated number of steps follows since the head is centered immediately left of the square containing tag " $]_{i+1}$ " for the first time after executing $A(>, i)$, which takes $T_{A}(i)$ steps. To clean up some final details: we can get rid of the fat head, covering 14 squares and sometimes shifting its center two squares in a single step, by cutting out a piece of tape of 14 squares and buffering it in the finite control. The remains of the tape are glued together and the contents of the buffered piece are swapped from the buffer to the scanned tape square and vice versa, according to the desired head motion, cf. the speed-up technique in [3].

On the required bits. Although the preceding simulation and its proof may not seem easy, the algorithm which does the work is pretty simple. As it happens, we are also frugal in the number of bits. On information-theoretical grounds we require about $k \log _{2} 2 n$ bits to represent any $k$-tuple of integers of absolute values up to $n$. In the exhibited simulation, we can use four bits for each digit of a count, need not more than $\log _{2} n$ digits for each count, and since there are but four tags, each tag can be encoded in two bits. Therefore, we use at most about $(4 k+2) \log _{2} n$ bits to represent $k$ counts of absolute values at most $n$. By restricting the most significant nonzero digit to absolute value 1 , and appropriately modifying the mappings UPDATE and INPUT, everything goes through as before but code $(c) \subseteq\{-2,-1,0,1,2,-\overline{1}, \overline{0}, \overline{1}\}^{\infty}, c \in \mathbb{Z}$. Thus we only have to use $(3 k+2) \log _{2} n$ bits to represent $k$ counts of absolute values at most $n$. Using only digits from $\{-2,-1,0,1,2, \overline{0}\}$ also suffices, but complicates the proof. How good a real-time algorithm is can be measured in the size of the storage alphabet used. Realizing that actual machines use a constant size storage alphabet, we observe that a large, although finite, storage alphabet in an algorithm implies a greater constant delay. That is, the reverse of a speed-up by decreasing the alphabet size. At the cost of a deterioration of the constant delay, implicit in the real-time solution presented, we can do better than using $(3 k+2) \log _{2} n$ bits. Using in $\S \S 2.1$ and 2.2 an analogous redundant symmetric $r$-ary representation, based on the digits $-r,-r+$ $1, \cdots,-1,0,1,2, \cdots, r-1, r$, we can get the bit count down to about $(1+$ $\left.4 / \log _{2} r\right) k \log _{2} n$ bits for maintaining $k$ counts of absolute value at most $n$. The implicit 
constant delay, however, rises proportional to $\log r$. In the limit, for $r \rightarrow \infty$, we achieve about the information-theoretical minimum in bits, but the constant delay goes to infinity, that is, it takes infinite time to execute a single step.

Note, however, that for no fixed finite storage alphabet a real-time simulation of but a single counter on an oblivious multitape Turing machine can reach the information-theoretical bit minimum. Such a simulation must use $\Omega(\log n)$ size representations for counts of size $n$, and we can argue that for each $n$ there must be at least $\log n$ representations. Hence we use at least $\log _{2} 2 n+\log _{2} \log n$ bits per count.

On the size of the fat head. In the simulation a head covering 10 squares suffices, which can be shown by a slight complication of the proof. Also, the head shift in a single step of $M$ need not exceed one square.

On the initially zero counts. As argued subsequent to the proof of Proposition 3, the assumption of initially zero counts is not essential. The theorem holds also for multicounter machines with each count initialized to an arbitrary integer.

3. Conclusion. For various theoretical and practical reasons, multitape Turing machines, restricted in one or more resources, serve as a standard against which to calibrate the power of other devices, or to compare the power among themselves under different resource restrictions. The commonly considered resources are time, space, number of tapes/storage heads and oblivious versus nonoblivious. The present simulation is, perhaps, the first one which is optimal in all of these resources at once: the use of no resource can be improved by relaxing the other resource restrictions. Apart from the fact that the simulating device is real-time, oblivious and uses but a single storage head, it is worthwhile to recall that there do not exist on-line Turing machines using $S(n) \in o(\log n)$ space, $S(n)$ unbounded [4]. Thus, the simulation is performed by the simplest (with respect to the considered resources) Turing machine which is not an outright finite automaton. Another resource, which is sometimes considered, is the number of head reversals. Again, it is easy to see that each multitape Turing machine needs, in the worst case, a linear number of head reversals to on-line simulate a counter machine, as does the presented simulation. (Although a multihead Turing machine can simulate a multicounter machine without head reversals [8], the simulation of such a device by a multitape Turing machine needs a linear number of head reversals.)

Some immediate applications. In a computation using $k$ stacks we may want to keep track of which pairs out of the $k$ stacks are of equal height at any time. Without slowing down the computation, we formerly needed $k-1$ stacks for doing so. Using the present method we need but one extra oblivious one-head tape unit, or two extra oblivious pushdown stores. A single pushdown store does not suffice. Similarly, we can keep track of the headpositions in multihead Turing machine computations.

Number representations. The reader may appreciate the following comment of John Locke on the intimate relation between counting and number representation.

For he that will count 20 , or have any idea of that number, must know that 19 went before, with the distinct name or sign of every one of them, as they stand marked in their order; for wherever this fails, a gap is made, the chain breaks, and the progress in numbering can go no further. So that to reckon right, it is required: (1) that the mind distinguish carefully two ideas, which are different one from another only by the addition or subtraction of one unit; (2) that it retain in memory [a systematic method for deriving] the names or marks of the several combinations, from a unit to that number, and that not confusedly and at random, but in that exact order that the numbers follow one another; in either of which, if it trips, the whole business of numbering will be disturbed, and there will remain only the confused idea of multitude, but the ideas necessary to distinct numeration will not be attained to. 
The one and only basic reason to denote numbers at all is for the purpose of comparing them, of whether the one is greater than the other, for without this capability no arithmetic is possible and with it all arithmetic is possible. Thus we must be able to distinctly represent all numbers, and if we have representations for all numbers up to a given one, then we must be able to derive the next one, or previous one, from the given one, while having a designated point of reference or benchmark number. This is the task expressed in the notion of a counter machine, and multicounter machines enable us to do arithmetic. The exhibited optimal implementation embodies a new representation for multituples of integers suitable for exercising that basic activity using minimal resources. Thus, for each $n=\left(n_{1}, n_{2}, \cdots, n_{k}\right) \in \mathbb{Z}^{k}, k \geqq 1$, each such representation for $n$ consists of a linear string of symbols, and is about as compact as possible. Such a representation has a distinguished access position $p$, and by considering only the three symbols centered on the access position we can

(i) add any vector $\delta=\left(\delta_{1}, \delta_{2}, \cdots, \delta_{k}\right) \in\{-1,0,1\}^{k}$ to $n$ to obtain such a representation for $n+\delta$;

(ii) for all $i, 1 \leqq i \leqq k$, determine whether $n_{i}+\delta_{i}=0$;

(iii) determine the new access position $p^{\prime} \in\{p-1, p, p+1\}$, which is also independent of $n$ and $\delta$. In $m$ successive additions the distance between the leftmost and rightmost intermediate access pointer positions is $O(\log m)$, for all $m>1$.

Note that Gray codes, as representations of integers, have vaguely similar properties for the case $k=1$. There, the representation of $n \pm 1, n \in \mathbb{Z}$, can be obtained from the representation of $n$ by changing a single symbol. However, the symbol in the representation which must be changed to obtain $n+1$ from $n$ can lie arbitrary far from the symbol which must be changed to obtain $n-1$ from $n$. Moreover, these positions depend on $n$ and whether we add or subtract, and do not allow us to test $n$ for 0 . The representation derivable from the simulation in [1] is closer to the one above, for the case $k=1$, but the new access position $p^{\prime}$ in (iii) depends on $n$ and $\delta$. None of these representations have any of the properties (i)-(iii) in case $k>1$.

Augmented counter machines. Apart from the basic one-step multicounter operations, several other one-step operations can be synthesized using concealed auxiliary counters, such as tests for equality amongst counters (by maintaining all differences on auxiliary counters). It is known [2] that the operations "set counter $i$ to zero" or "set counter $i$ to the value of counter $j$ " $(i \neq j)$ cannot be synthesized as one-step operations on a multicounter machine. At the end of $\S 2.2$ we noted that the requirement of initially zero counters was not essential for the present simulation. It can be proved [9] (this issue, pp. 34-40) that with a suitable embellishment the present simulation can also support the one-step operation "set counter $i$ to the value of counter $j$ " $(i \neq j)$. Define an augmented counter machine (ACM) just like a multicounter machine but with the one-step input operations "set counter $i$ to the value of counter $j$ " (for any pair of counters $i, j$ ) added and any initial counter contents in $\mathbb{Z}$ allowed. Such a machine can execute quite powerful instructions in one step. For example:

$$
L: \text { if }(x<y \& y \geqq c) \text { then }(x \leftarrow z ; z \leftarrow d) \text { else }\left(x \leftarrow y \text {; goto } L^{\prime}\right) \text { fi }
$$

with $x, y, z$ integer variables, $c, d$ integers and $L, L^{\prime}$ labels, is a one-step instruction for an ACM.

THEOREM. Each augmented counter machine can be real-time simulated by an oblivious one-head tape unit in logarithmic space.

Uniform space complexity. Viewed in space-time, the bracket manipulator head describes an interesting curve. This is perhaps best expressed by stating that the 
two-dimensional space-time trajectory described by the center of the greatest tape segment, delimited by brackets with indices $j, j^{\prime} \leqq i$, is the same as that described by the center of the greatest tape segment, delimited by brackets with indices $j, j^{\prime} \leqq i-1$, $i>1$, subsequent to multiplying the time scale of the latter by $S(i) / S(i-1)$ and the space scale of the latter by $i /(i-1)$. This shows that the number of distinct squares visited in each time interval of $n$ steps, for all $n \geqq 1$, is $\Theta(\log n)$. Generalizing this observation, we say that a multitape Turing machine $M$ uses uniform logarithmic space if, for any unbounded input sequence, the total number of distinct squares, visited on $M$ 's storage tapes, for each interval of $n$ steps, for all $n \geqq 1$, is $O(\log n)$. It can be shown [10] that each multitape Turing machine using uniform logarithmic space can be real-time simulated by an oblivious one-head tape unit using uniform logarithmic space.

Oblivious simulations. It seems to us that also the converse of the maxim leading to Proposition 1 holds generally. Viz., if we can simulate arbitrarily many storage devices by a fixed number of, possibly different, devices then we can do so obliviously retaining the same resource bounds. The point here is that if the multitude of head movements of an arbitrary number of heads can be accommodated by the motion of a fixed number of heads, then there is no reason to suppose that any trajectory of the latter can make significant use of particular input streams.

\section{REFERENCES}

[1] M. J. FISCHER AND A. L. ROSENBERG, Real-time solutions of the origin-crossing problem, Math. Systems Theory, 2 (1968), pp. 257-263.

[2] P. C. Fischer, A. R. MEyer And A. L. Rosenberg, Counter machines and counter languages, Math. Systems Theory, 2 (1968), pp. 265-283.

[3] J. Hartmanis AND R. E. STEARnS, On the computational complexity of algorithms, Trans. Amer. Math. Soc., 117 (1965), pp. 285-306.

[4] J. E. HopcrofT AND J. D. Ullman, Some results on tape-bounded Turing machines, J. ACM, 16 (1969), pp. 168-177.

[5] M. MINSKY, Recursive unsolvability of Post's problem of 'tag' and other topics in the theory of Turing machines, Ann. Math., 74 (1961), pp. 437-455.

[6] N. PIPPENGER AND M. J. FisCHER, Relations among complexity measures, J. ACM, 26 (1979), pp. 361-381.

[7] C. P. SCHNORR, The network complexity and Turing machine complexity of finite functions, Acta Informatica, 7 (1976), pp. 95-107.

[8] P. M. B. VITÁNYI, On efficient simulations of multicounter machines, Inform. and Control, 55 (1982), pp. 20-39.

[9] - An optimal simulation of counter machines: the ACM case, this Journal, this issue, pp. 34-40.

$[10]-$ On the simulation of many storage heads by one, Technical Report IW 228, Mathematisch Centrum, Amsterdam, 1983, preliminary version in Proc. 10th ICALP, Lecture Notes in Computer Science 154, Springer-Verlag, Berlin, 1983, pp. 687-694. 